\title{
Numerical studies of shock wave interactions with a supersonic turbulent boundary layer in compression corner:Turning angle effects
}

\author{
Fulin Tong ${ }^{\mathrm{a}}$, Changping Yu ${ }^{\mathrm{b}}$, Zhigong Tang ${ }^{\mathrm{a}}$, Xinliang $\mathrm{Li}^{\mathrm{b}, \mathrm{c}, *}$ \\ ${ }^{a}$ Computational Aerodynamics Institute, China Aerodynamics Research and Development Center, Mianyang 621000, China \\ ${ }^{\mathrm{b}}$ LHD, Institute of Mechanics, Chinese Academy of Sciences, Beijing, 100190, China \\ ' School of Engineering Science, University of Chinese Academy of Sciences, Beijing, 100049, China
}

\section{A R T I C L E I N F O}

\section{Article history:}

Received 14 September 2016

Revised 24 February 2017

Accepted 8 March 2017

Available online 10 March 2017

\section{Keywords:}

Compression corner

Low-frequency oscillation

Coherent vortex structure

Power spectrum

Turbulent kinetic energy budget

Direct numerical simulation

\begin{abstract}
A B S T R A C T
Direct numerical simulations (DNS) were performed to investigate the interactions of a Mach 2.9 turbulent boundary layer with shock waves of varying strengths in compression corner. The supersonic turbulent boundary layer was triggered by wall blowing-and-suction perturbations. The shock waves were produced by two-dimensional compression corners of 8, 14, 20 and $24^{\circ}$. Compared with previous DNS results and experimental data, the numerical calculations were validated. The effects of shock wave on the boundary layer are studied by both flow visualizations and statistical analysis, and the results show that the intensity of fluctuations is amplified greatly by the shock wave. With the increasing of turning angle, three-dimensionality of separation bubble is significantly enhanced. Based on the statistics and power spectrum of the wall pressure signals, the effect of turning angle on the unsteadiness of shock motion is also studied, and the results show that the shock motions are quite different in the small and the large turning angle cases. The motion in the $8^{\circ}$ and $14^{\circ}$ cases is characterized by high-frequency and smallamplitude, but the low-frequency and large-scale streamwise oscillation is the main feature in the $20^{\circ}$ and $24^{\circ}$ cases. The effect of turning angle on the turbulence state is analyzed by using the anisotropy of Reynolds stress tensor. The coherent vortex structures are also studied qualitatively. The results indicate that the cane-like streamwise vortexes in the near-wall region are the dominant structure for the small angle cases, while the hairpin vortexes and packets in the outer layer play the leading role in the large angle cases. According to the quantitative analysis of turbulent kinetic energy budgets in the separation region, the effect of turning angle on the transport mechanism is studied. It is found that the influence of shear layer above separation bubble on the mechanism is significant.
\end{abstract}

(C) 2017 Elsevier Ltd. All rights reserved.

\section{Introduction}

Shock wave and turbulent boundary layer interaction (SWT$\mathrm{BLI}$ ) is a common flow phenomenon in high speed flow around aircrafts. The related complicate phenomena, including flow separation and reattachment, low-frequency unsteadiness and high local thermal loads, can significantly affect the aerodynamic performance of aircraft. According to the generation of shock wave, this problem can be simply classified into two canonical categories, which are compression corner and reflected shock. Although the interactions have been investigated extensively by experimental [1-7] and numerical [8-15] methods during the past decades, there still exist a few issues needed to be ascertained [16-18]. The separation shock oscillation, with characteristic frequency of one or two orders lower than the typical frequency of super-

\footnotetext{
* Corresponding author.

E-mail address: lixl@imech.ac.cn (X. Li).
}

sonic turbulent boundary layer, has always been a difficult and driving research area for several decades. The physical source of low-frequency unsteadiness is still in debate with two apparently opposite views, which are upstream and downstream mechanisms respectively. Comprehensive reviews on this subject have been given by Clemens et al. [17]

The experiments of compression corner configuration covered wide range of turning angles and Reynolds numbers. Ardonceau et al. [1] used laser and hotwire velocimeters to investigate the Reynolds stress tensor through the compression corner. The shear component was found to rise more rapidly than other components. Turbulence amplifications induced by shock interactions were discussed by Smits \& Muck [2] using the experimental data. It is proposed that the shock unsteadiness might be the main reason to explain the difference between their experiments and rapid distortion analysis. Dolling \& Or [3] measured the wall-pressure fluctuation at different streamwise locations in the compression corner. Intermittency of shock motion was revealed by wall pres- 
sure signals and the length scale of intermittent region increased with ramp angle. To this day, unsteadiness and low frequency of shock motion has become an increasingly important hot topic in SWTBLI problem. Beresh [4] found a correlation between the moving shock and velocity fluctuations in upstream turbulent boundary layer. However, Erengil \& Dolling [5] proposed that the pressure fluctuations might be the cause of low frequency motion. Ganapathisubramani et al. [6] also investigated the correlation between the superstructures of turbulent boundary layer and shock motion. In summary, the available experimental data of interactions in compression corner are numerous, but most experiments were under relatively high Reynolds numbers, which are not accessible for DNS. Recently, in order to verify the DNS results, Bookey et al. [7] performed a series of SWTBLI experiments under low Reynolds numbers.

With the fast development of computational techniques and super-computers, flow mechanisms of shock wave and supersonic turbulent boundary layer interactions have been extensively studied by large-eddy simulation (LES) and DNS. Touber et al. [8] performed LES studies of different reflection configurations. The relation between shock strength and the interaction length, which was firstly inferred from previous experimental data, was confirmed by their LES data. Moreover, the unsteady results of all tested cases had also shown evidence of the presence of lowfrequency shock motions. The effects of shock strength on SWTBLI were studied by Morgan et al. [9] using LES. With the increment of the interaction strength, the separation region expanded and the low-frequency peak energy shifted toward lower frequencies. Rizzetta et al. [10] used LES with dynamic Smagorinsky model to study the supersonic compression corner with varying ramp angles. The time-averaged wall pressure and skin friction showed good agreement with experimental data.

The first and well-known DNS of compression corner was reported by Adam [11]. The shock motion and turbulent fluctuations had been studied delicately in the interaction region. It was found that the oscillation frequency of shock is similar to the bursting frequency of incoming turbulent boundary layer. However, his numerical results cannot be compared directly with the experimental data because of the limited Reynolds number. In recent years, $\mathrm{Wu}$ et al. [12-13] and Priebe et al. [14] performed DNS studies of a Mach number of $2.9,24^{\circ}$ compression corner to analyze the shock motion. The inflow free stream is similar to the experiment of Bookey et al. [7], which is at the accessible Reynolds number for DNS on current conditions. Satisfactory comparisons in the mean and fluctuating results were reported. And the low frequency streamwise oscillation motion of separation shock was inferred from the power spectral analysis of wall-pressure signals. Furthermore, the correlations among the separation point, reattachment point and shock location were studied systematically by Wu et al. [13], indicating that the downstream flow is the main determinants of low-frequency shock unsteadiness. Priebe et al. [14] investigated the low-pass filtered instantaneous flow fields and conjectured that an inherent instability in the downstream separated bubble is the physical origin. In addition, Li et al. [15] compared the DNS data of laminar and turbulent calculations of the same compression corner with similar inflow conditions, which is also likewise the experiment of Bookey et al. [7]. The inlet turbulence was triggered by blowing and suction, on the other hand, no perturbation was added in the laminar simulation. However, the low frequency oscillation phenomenon is found in both results. It is suggested that the upstream turbulent disturbance has little relation with the low frequency motion of unsteady shock.

In our previous research [35], the authors have studied the effects of wall temperature on the length of separation bubbles in a 24-degree corner. In this paper, the main objectives are to examine SWBLI under various shock wave strengths and to

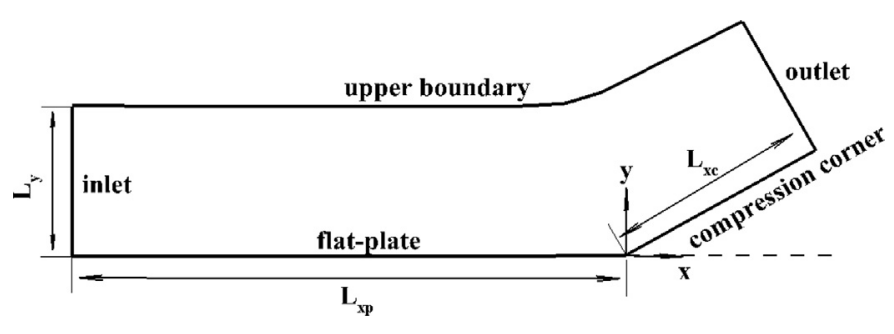

Fig. 1. Illustration of computational model.

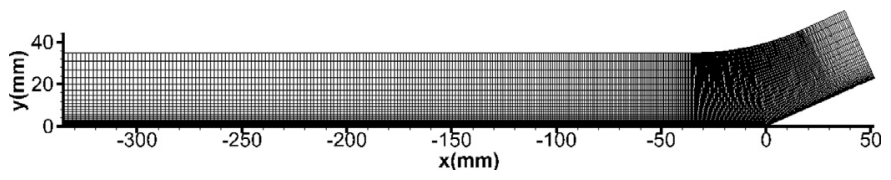

Fig. 2. Illustration of computational grid for compression corner.

improve the basic understanding of SWBLI by a detailed analysis of new DNS data. The configuration chosen here is a separated compression corner which has many similarities with the reflected shock problems. Four different corner angles, 8, 14, 20 and 24\%, are considered. The Mach number of incoming boundary layer is fixed at 2.9. Additionally, for better comparisons, the selected flow parameters are similar with the experiment of Bookey et al. [7] and DNS of Wu et al. [12]. The present DNS is performed using a band-optimized weighted essentially non-oscillatory (WNEO) methods [19] with limiting technique [12]. The setup of this DNS is presented in Section 2, including numerical methods, computational grid and generation of turbulent inflow. The accuracies of present DNS data, validated by comparison against experimental data and previous DNS results, reported in Section 3. At last, the DNS results of this study and discussions are present in Section 4.

\section{Computational setup}

\subsection{Numerical methods}

The governing equations are the non-dimensionalized conservative form of Navier-Stokes equations in curvilinear coordinate

$$
\frac{\partial U}{\partial t}+\frac{\partial\left(F_{c}+F_{v}\right)}{\partial \xi}+\frac{\partial\left(G_{c}+G_{v}\right)}{\partial \eta}+\frac{\partial\left(H_{c}+H_{v}\right)}{\partial \zeta}=0
$$

where $t$ denotes the time, $\xi, \eta$, and $\zeta$ are the computational coordinates. $U$ is the conservative variables. $F_{c}, G_{c}$, and $H_{c}$ are the convective flux terms in the $\xi, \eta$, and $\zeta$ directions, respectively. $F_{v}$, $G_{v}$ and $H_{v}$ are the corresponding viscous flux terms. Perfect gas is selected as working fluid.

A high-resolution CFD package, OpenCFD-SC, is used to perform the DNS calculation in this study. The package has already been applied to a wide range simulation of compressible turbulent flow, such as acoustic calculation for supersonic turbulent boundary [20], supersonic and hypersonic flat-plate flow [21] and hypersonic boundary layer transition over blunt cone [22].

The convective flux terms are computed by the Steger-Warming splitting method and the optimized WENO scheme of Martin et al. [19]. To maintain maximum order of accuracy and bandwidth, the optimized scheme is designed with symmetry stencils as ordinary eighth-order central scheme. Therefore, the numerical dissipation of this scheme is less than original WENO scheme. In addition, a limiting technique reported by $\mathrm{Wu}$ et al. [12] is used to proceed the weighting calculation, further minimizing the computation cost and translating the optimized WENO to linear scheme. These numerical methods have been widely validated with great success in DNS studies of shock wave and turbulent boundary layer interaction, especially in the cases with large separation [12-14]. Viscous 

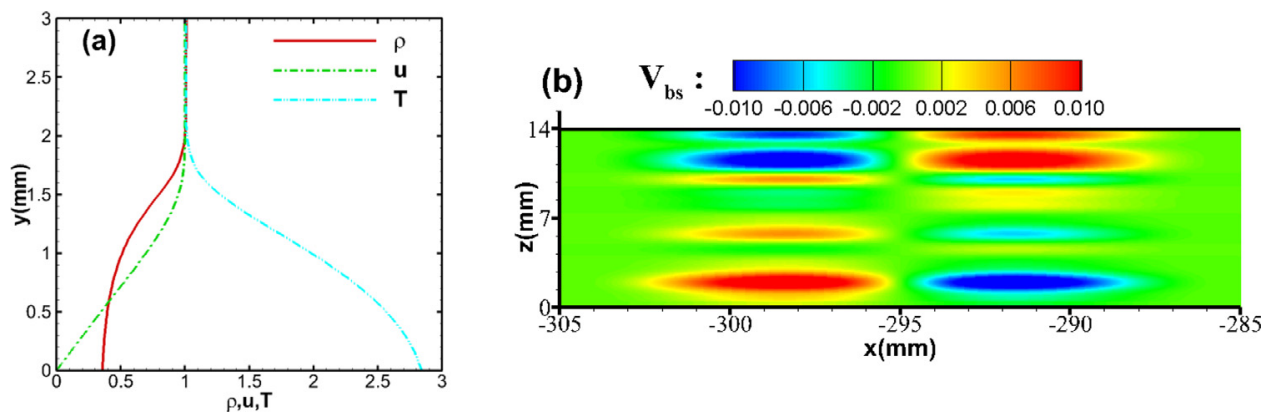

Fig. 3. Laminar boundary layer profile (a) and contour of the normal velocity component $\mathrm{V}_{\mathrm{bs}}$ in the $x-z$ plane (b).

Table 1

Inflow free-stream and turbulent boundary layer parameters at the reference point.

\begin{tabular}{|c|c|c|c|c|c|c|c|c|}
\hline \multirow[t]{2}{*}{ Case } & \multicolumn{4}{|c|}{ Inflow free-stream } & \multicolumn{4}{|c|}{ Turbulent boundary layer parameters } \\
\hline & $\mathrm{Ma}_{\infty}$ & $R e_{\infty}$ & $\mathrm{T}_{\infty}(\mathrm{k})$ & $\mathrm{T}_{\mathrm{w}}(\mathrm{k})$ & $\theta(\mathrm{mm})$ & $\delta^{*}(\mathrm{~mm})$ & $\delta(\mathrm{mm})$ & $c_{f}$ \\
\hline Present DNS & 2.9 & 5581.4 & 108.1 & 307.0 & 0.41 & 2.06 & 6.5 & 0.00256 \\
\hline Bookey et al. [7] & 2.9 & 5581.4 & 108.1 & 307.0 & 0.43 & 2.36 & 6.7 & 0.00225 \\
\hline Wu et al. [12] & 2.9 & 5581.4 & 108.1 & 307.0 & 0.38 & 1.80 & 6.4 & 0.00217 \\
\hline
\end{tabular}

flux terms are directly computed by the ordinary eighth-order central scheme. Explicit third-order total variation diminishing (TVD) type Runge-Kutta method is used for the time marching.

\subsection{Computational model and grids}

The computational model, as shown in Fig. 1, contains two parts. The first part is a flat-plate domain, and the second part is a compression corner with four different turning angles, which are $8^{\circ}, 14^{\circ}, 20^{\circ}$ and $24^{\circ}$ respectively. The $x$ - and $y$ - coordinates are shown in Fig. 1 and the $z$-coordinate are spanwise coordinate. The coordinate origin is located at the corner region of the ramp. The dimensions of computational domain are also shown in this figure. For all the four cases, the domain is divided into two parts in the streamwise direction. The domain of the flat-plate $L_{x p}$ extends from $-335 \mathrm{~mm}$ to $0 \mathrm{~mm}$, which measured from the computational inlet to coordinate origin. The streamwise length $L_{x c}$ along the compression corner surface is $50 \mathrm{~mm}$. The spanwise dimension $L_{z}$ is $14 \mathrm{~mm}$ and the wall-normal dimension $L_{y}$ is $35 \mathrm{~mm}$.

To minimize numerical errors in the calculation of Jacobian matrices, computing grids are generated using analytical transformations [12]. As shown in Fig. 2, the number of grid points for all considered cases is $2160 \times 160 \times 140$ (streamwise $\times$ wallnormal $\times$ spanwise), which are concentrated in the corner region $(-35 \mathrm{~mm} \leq \boldsymbol{x} \leq 35 \mathrm{~mm})$ and near-wall region in the wall-normal direction. The grids are equally spaced in the spanwise direction. In wall units, based on the turbulent boundary layer properties taken at the reference point $(\boldsymbol{x}=-35 \mathrm{~mm})$, the grid spaces in the separation region of ramp corner are $\Delta \mathrm{x}^{+} \approx 4.5, \Delta \mathrm{y}_{\mathrm{w}}{ }^{+} \approx 0.5$ and $\Delta z^{+} \approx 5.0$, which are much smaller than previous DNS of flat-plate supersonic turbulent boundary layer [21].

\subsection{Initial and boundary conditions}

The initial conditions and inlet boundary conditions are prescribed as follows. Two-dimensional laminar results, obtained with the same grid and inflow conditions as the turbulent calculations, are selected as the initial conditions. The corresponding laminar profile is selected as the inlet boundary condition, which will be shown in Fig. 3a. At the outlet and upper boundary, a sponge layer is applied to avoid the reflection of disturbance wave. No-slip and adiabatic wall conditions are used on the corner surface. In the spanwise direction, periodic boundary condition is used.

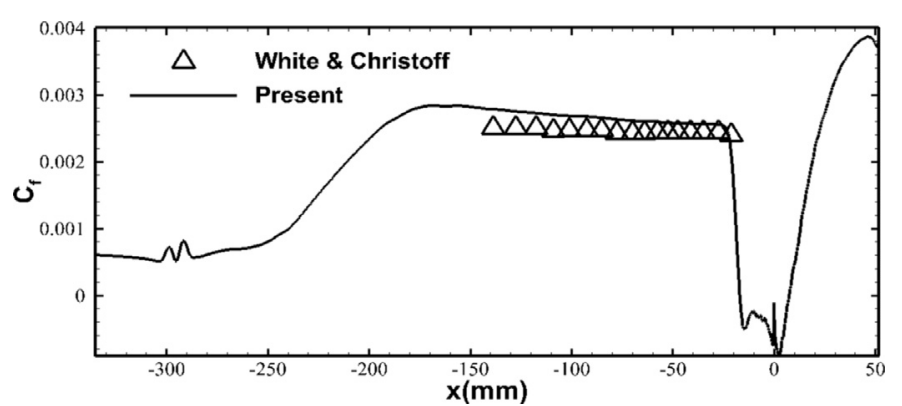

Fig. 4. Distribution of wall skin-friction coefficient in the $24^{\circ}$ compression corner.

\subsection{Turbulent inflow generation}

In the present simulation, a flat-plate region with blowing-andsuction pertubation is used to generate the turbulent flow as the inflow of the corner region. The blowing-and-suction velocity $\boldsymbol{v}_{\boldsymbol{b s}}$ is set as [23]

$$
\begin{aligned}
v_{b s} & =A f(x) g(z) h(t) \\
f(x) & =4 \sin (\theta)[1-\cos (\theta)] /(27)^{1 / 2}, \theta=2 \pi\left(x-x_{a}\right) /\left(x_{b}-x_{a}\right) \\
g(z) & =\sum_{l=1}^{l_{\max }} Z_{l} \sin \left[2 \pi l\left(z / z_{\max }+\phi_{l}\right)\right], \sum_{l=1}^{l_{\max }} Z_{l}=1, Z_{l}=1.25 Z_{l+1} \\
h(t) & =\sum_{m=1}^{m_{\max }} T_{m} \sin \left[2 \pi m\left(\beta t+\phi_{m}\right)\right], \sum_{m=1}^{m_{\max }} T_{m}=1, T_{m}=1.25 T_{m+1}
\end{aligned}
$$

where $\boldsymbol{x}_{a}$ and $x_{b}$ indicate the beginning and end location of the blowing and suction zone, respectively. $Z_{\max }$ is the spanwise length of computational domain. $\boldsymbol{A}$ is the amplitude of disturbance and $\boldsymbol{\beta}$ is the fundamental frequency of disturbance. $\Phi_{l}$ and $\Phi_{m}$ are the random numbers ranging between 0 and 1 . In the current simulation, $\boldsymbol{A}=0.2$ and $\boldsymbol{\beta}=0.1$.

Fig. 3 presents the laminar boundary layer profile at the inlet boundary of computational domain, and it also shows the normal velocity component $\boldsymbol{v}_{\boldsymbol{b s}}$ in blowing and suction region of flat-plate. As shown in Fig. $3 \mathrm{~b}$, the normal velocity in the $x-z$ plane is asymmetric in the spanwise direction. Eventually, the asymmetry results in the generation of near-wall streamwise vortices [21].

The mean wall skin-friction coefficient in the $24^{\circ}$ case is shown in Fig. 4. The mean variables reported in this paper are obtained 

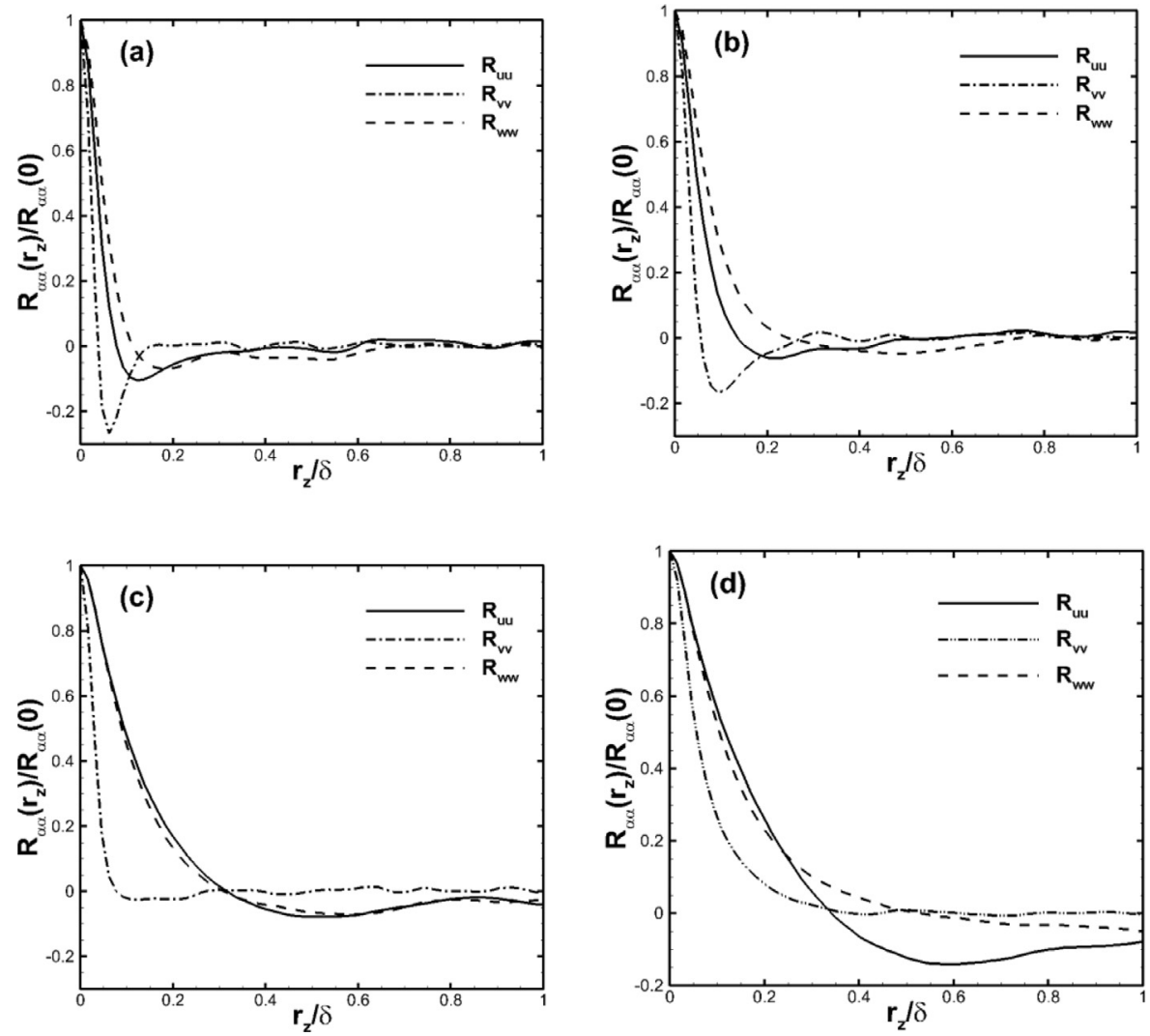

Fig. 5. Two-point correlations of the velocity. (a) $\mathrm{x} / \delta=-5.4, \mathrm{y}^{+}=5$; (b) $\mathrm{x} / \delta=-5.4, \mathrm{y}^{+}=10 ;$ (c) $\mathrm{x} / \delta=-1.2, \mathrm{y}^{+}=5 ;(\mathrm{d}) \mathrm{x} / \delta=0, \mathrm{y} / \delta=0.3$.

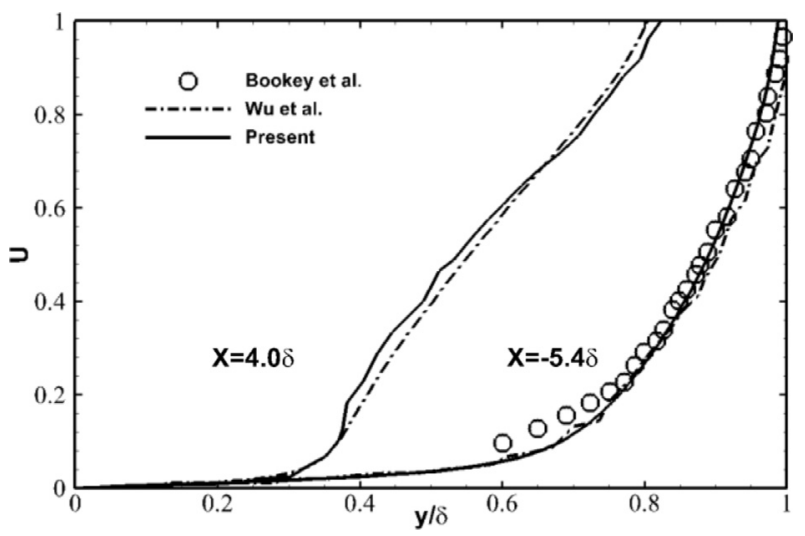

Fig. 6. Mean velocity profiles at $\mathrm{x}=-5.4 \delta$ and $4.0 \delta$. Solid lines: present DNS; dashed lines: DNS, Wu et al. [12]; Circles: experiment, Bookey et al. [7].

by averaging in time and the homogeneous spanwise direction. Fig. 4 also plots the theoretical estimation for the fully turbulent regime, which is given as [36]

$$
\begin{gathered}
C_{f}=\frac{0.455}{S^{2}}\left[\log \left(\frac{0.06}{S}\left(\operatorname{Re}_{x}-\operatorname{Re}_{x_{0}}\right) \frac{\overline{\mu_{e}}}{\overline{\mu_{w}}} \sqrt{\frac{\overline{T_{e}}}{\overline{T_{w}}}}\right)\right]^{-2} \\
S=\frac{1}{\arcsin A} \sqrt{\frac{\overline{T_{w}}}{\overline{T_{e}}}-1 \quad A=\left(\frac{\gamma-1}{2} M_{e}^{2} \frac{\bar{T}_{e}}{\overline{T_{w}}}\right)^{1 / 2}}
\end{gathered}
$$

where subscript $\boldsymbol{e}$ and $\boldsymbol{w}$ refer to properties at the outer edge of the boundary layer and the wall, respectively. The calculation results agree well with the above theoretical correlation in the fully developed turbulent region. The mean skin friction coefficient sig-

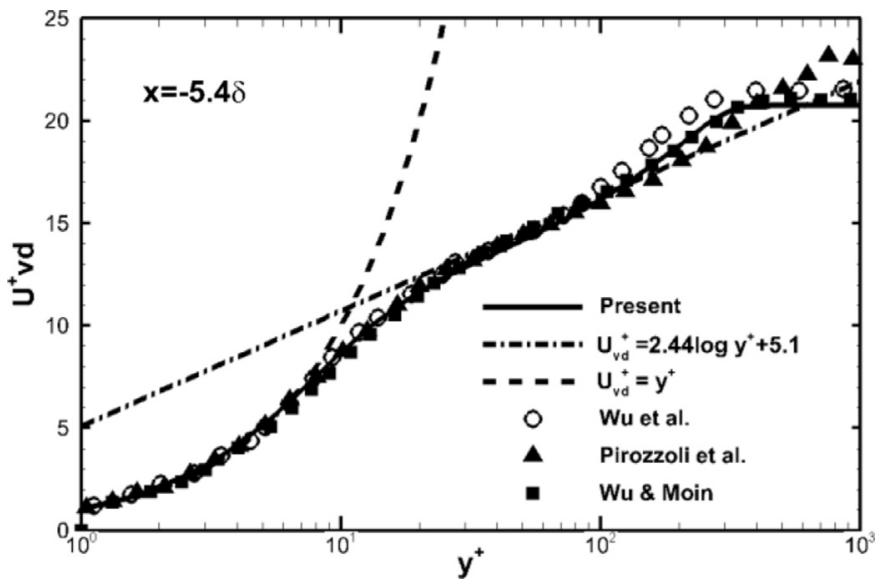

Fig. 7. Van Direst transformed mean velocity profiles at the reference point $(\mathrm{x}=-5.4 \delta)$. Solid line: present DNS; open circle: Wu et al. [12]; triangles: Pirozzoli [23]; square: Wu \& Moin [24].

nificantly rises up at the region $-280 \mathrm{~mm} \leq \mathrm{x} \leq-180 \mathrm{~mm}$, indicating the onset of transition. As the boundary layer develops into full turbulence, the mean skin friction coefficient gradually approaches the theoretical estimation. According to the analysis of Gao \& $\mathrm{Li}$ [21], the type of this triggered transition is bypass transition.

The obtained turbulent boundary layer parameters at the reference point are listed in Table 1 , where $\boldsymbol{\theta}, \boldsymbol{\delta}^{*}, \boldsymbol{\delta}$ and $\boldsymbol{C}_{\boldsymbol{f}}$ denote the boundary layer momentum thickness, the boundary layer displacement thickness, the boundary layer thickness and wall skin-friction coefficient, respectively. For comparison, the boundary layer parameters of Bookey et al. [7] and $\mathrm{Wu}$ et al. [12] are also given in this table. It is obvious that the fully developed turbulence 

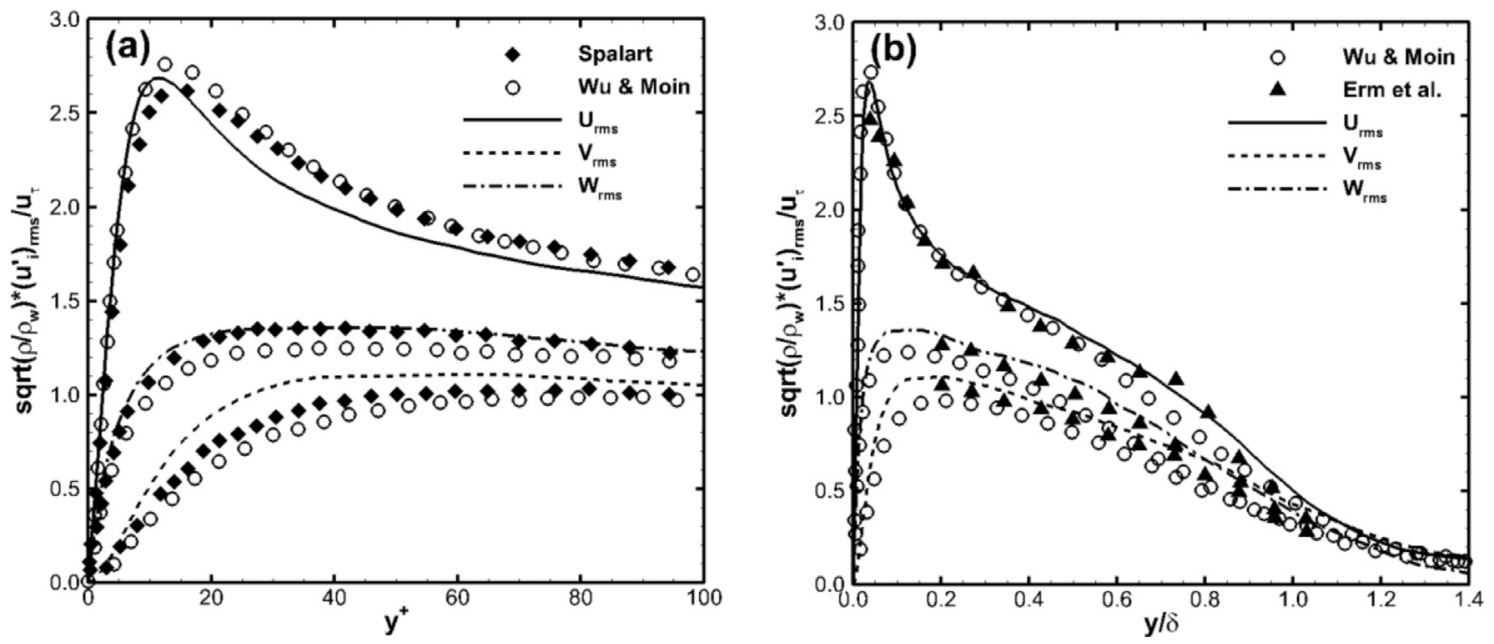

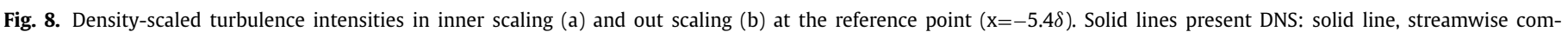

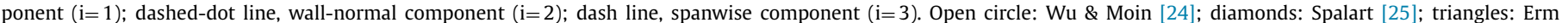
[26].
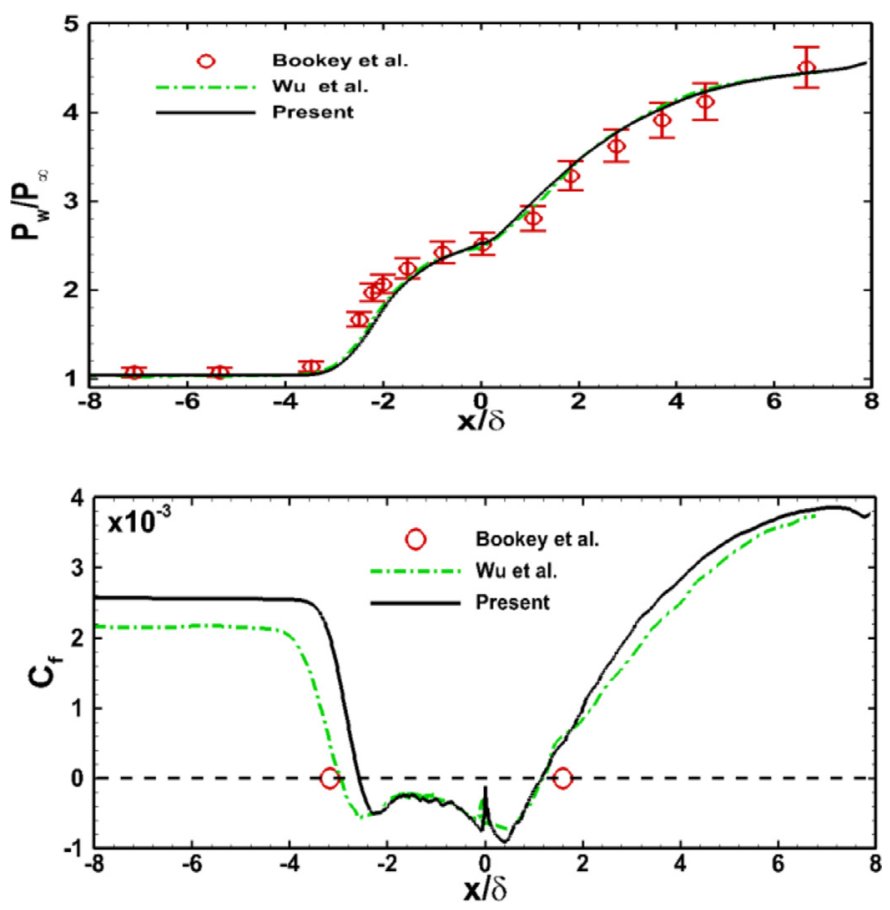

Fig. 9. Distribution of mean wall pressure (a) and skin-friction coefficient (b). Solid lines: present DNS; dash-dot line: Wu et al. [12]; symbol: Bookey et al. [7].

in this paper are very close to experimental and previous DNS data, but the wall skin-friction coefficient of present simulation is slightly higher. The difference is likely caused by the methods of turbulence generation. In the previous DNS of Wu et al. [12], recycling-rescaling technique was used to generate the inlet turbulence. And in the experiments of Bookey et al. [7], incoming turbulent boundary layer was triggered by the cylindrical wire trip.

\section{Accuracy of DNS}

Statistics are collected after long time calculation when the flow becomes statistically equilibrium. The entire time of simulation in the $8^{\circ}$ and $14^{\circ}$ cases is $600 \delta / \mathrm{U}_{\infty}$. In order to study the low-frequency characteristic of unsteady shock motion, the entire time of simulation in the $20^{\circ}$ and $24^{\circ}$ cases is $3000 \delta / \mathrm{U}_{\infty}$. In this simulation, the boundary layer thickness $\delta$ is selected at the reference point and $U_{\infty}$ is the inflow velocity. Three-dimensional instantaneous flow fields are then saved every $1.23 \delta / \mathrm{U}_{\infty}$ and the total number is 500 . Additionally, wall-pressure signals along the median line through the interaction region are sampled every $0.06 \delta / \mathrm{U}_{\infty}$.

Assessments of the grid resolution in the spanwise direction are performed firstly. The auto-correlation functions are analyzed in Fig. 5. Here, the correlation coefficient is defined as [23]

$R_{\alpha \alpha}\left(r_{z}\right)=\sum_{K=1}^{N_{z}-1}\left\langle\alpha_{K} \alpha_{K+K_{r}}\right\rangle, \quad K_{r}=0,1, \ldots, N_{z}-1$

where $K_{r}$ is the number of grid points in the spanwise direction, and $\alpha$ represents the fluctuations of flow variables. This figure shows that, whether at flat-plate or the interaction region, the correlations for velocity components both decay towards zero after a distance larger than half of the spanwise domains size. It suggests that the computational domain in the spanwise direction is wide enough to resolve the large-scale structure in the interaction region.

In order to validate the results of $24^{\circ}$ compression corner, the mean and turbulence quantities, including the mean velocity profile, turbulence intensities, wall pressure and skin-friction coefficient, power spectral density of pressure fluctuations, are compared with experimental and previous DNS data.

Fig. 6 presents the mean velocity profiles at different streamwise location. The dashed lines denote previous DNS data [11]. The circles denote experimental data of Bookey et al. [7]. It is apparent that three profiles at the reference point agree well with each other. Downstream of the interaction region, velocity profiles of DNS are in good agreement. However, both of DNS results deviate from experimental data in the near-wall regions, where the errors of velocity measurement are significant. Fig. 7 plots the Van-Driest transformed mean velocity profile at the reference point. It is clear that the logarithmic behavior agrees well with the log-law. The density-scaled turbulence intensities at the reference point are shown in Fig. 8. It is obvious that reasonable agreement is obtained with incompressible DNS data and low-speed boundary layer experiments.

Fig. 9 depicts the streamwise distribution of the mean wall pressure and skin-friction coefficient through the interaction region. Evidently, the calculated wall pressure collapse to the DNS result of $\mathrm{Wu}$ et al. [12], both within the experimental uncertainty. 

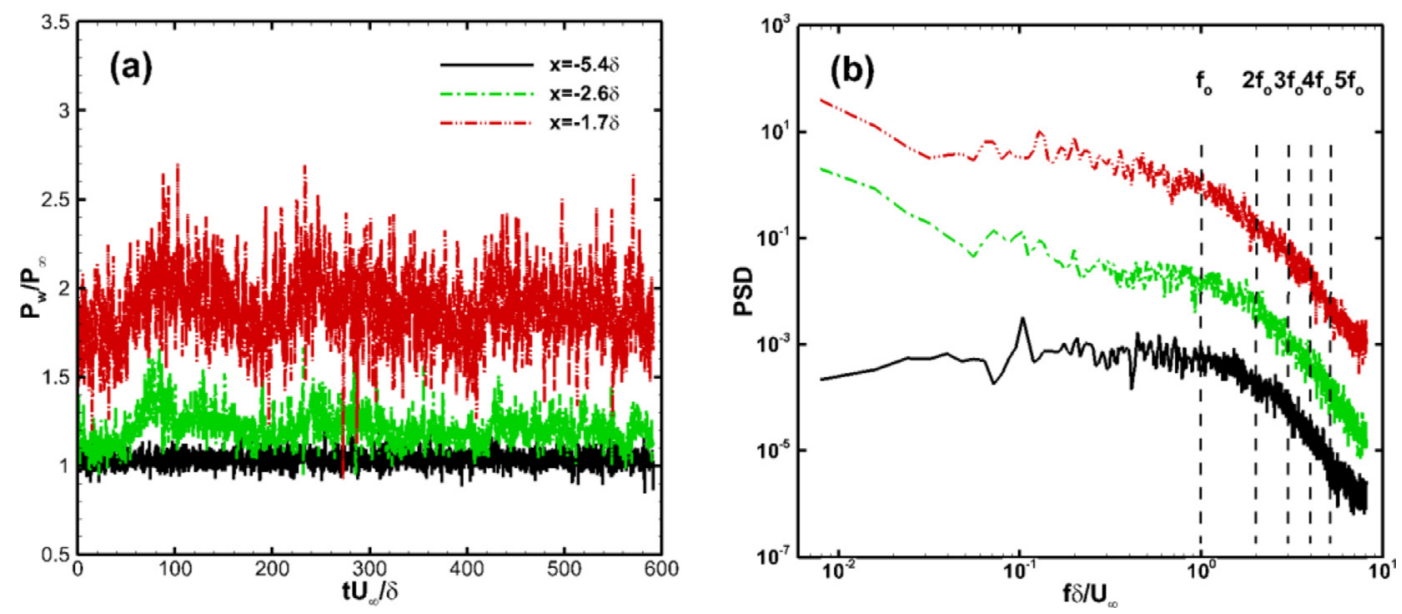

Fig. 10. Wall pressure signals (a) and corresponding power spectral density (b) at different streamwise locations.
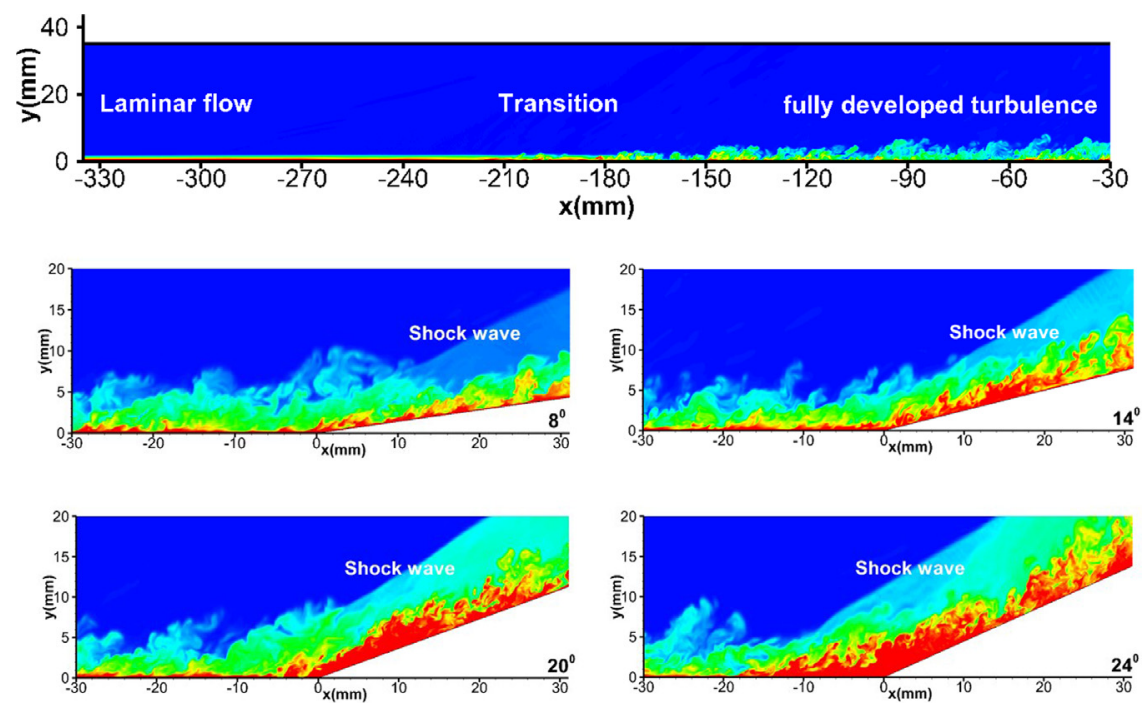

Fig. 11. Instantaneous temperature flow-field in midspan section $(\mathrm{z}=7 \mathrm{~mm})$. Contours: from $1.0 \mathrm{~T}_{\infty}$ to $2.5 \mathrm{~T}_{\infty}$.
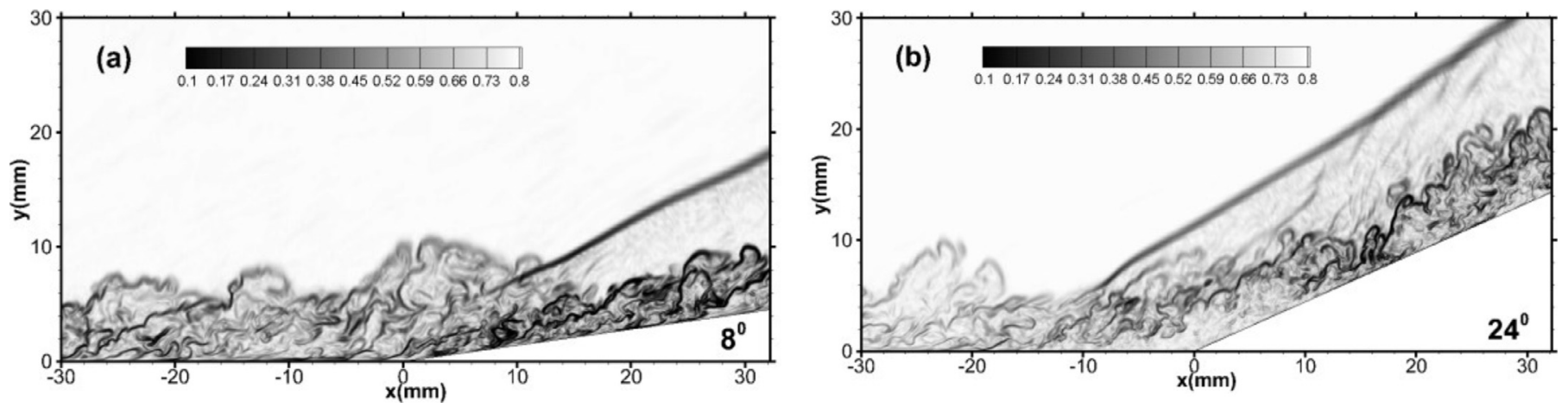

Fig. 12. Instantaneous numerical schlieren. (a) $8^{\circ}$; (b) $24^{\circ}$.

There is a rapid rise from the region $x / \delta=-3.5$, followed by a plateau farther downstream. As shown in Fig. 9, the separation point in current results is much closer to the ramp corner than that of $\mathrm{Wu}$ et al. [12]. The main reason for this difference might be that the wall skin-friction coefficient of incoming turbulent boundary layer in this paper is slightly higher, which inhibits the development of separation bubble. However, the distribution of skin-friction coefficient in the separation bubble and downstream the interaction is both in reasonable agreement with previous DNS results.

Fig. 10 shows the wall pressure signals and corresponding power spectral densities (PSD) at three different streamwise positions. The spectra for these signals are multiplied by different scales to avoid overlapping. It is clear that the peak frequency in the upstream undisturbed boundary layer is around $0.1-1.0 \mathrm{U}_{\infty} / \delta$, which is consistent with the characteristic frequency of turbulent 

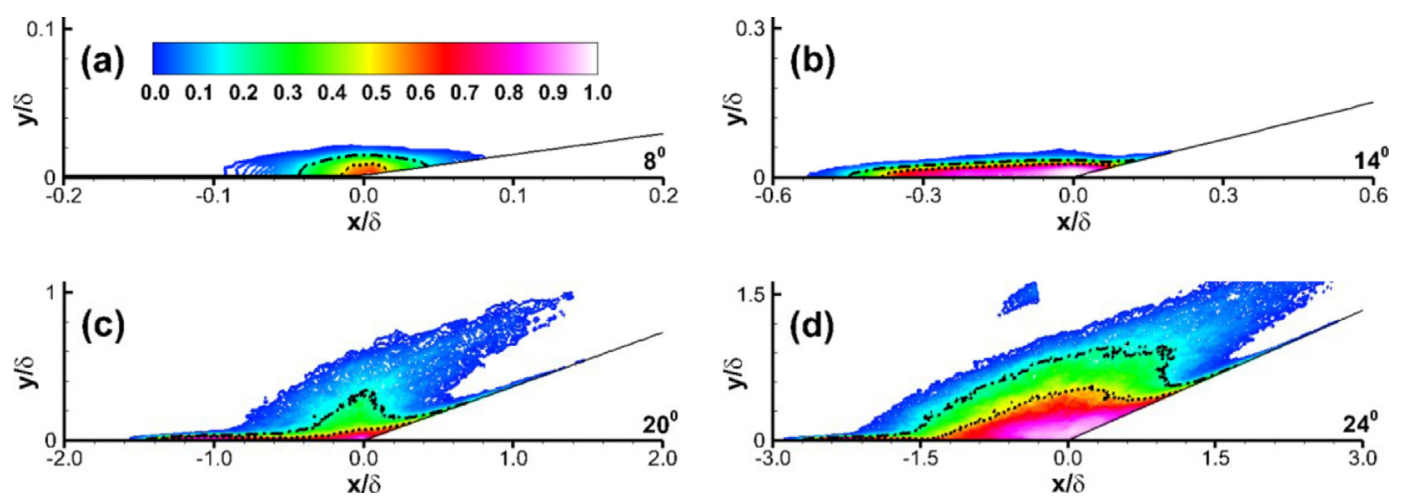

Fig. 13. Probability distribution of reversed flow. Dash line: $\gamma_{\mathrm{u}}=0.2$; dashed-dot line: $\gamma_{\mathrm{u}}=0.5$.

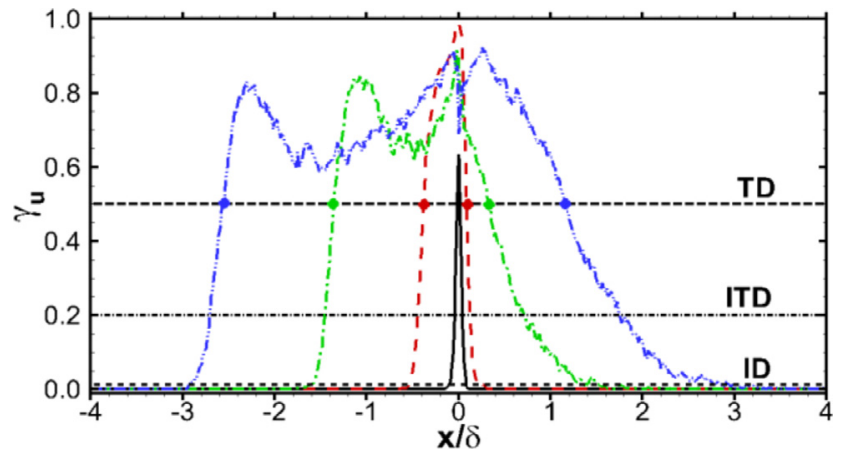

Fig. 14. Distribution of probability of wall points with separation. ID: incipient detachment; ITD: Intermittent transitory detachment; TD: transitory detachment.

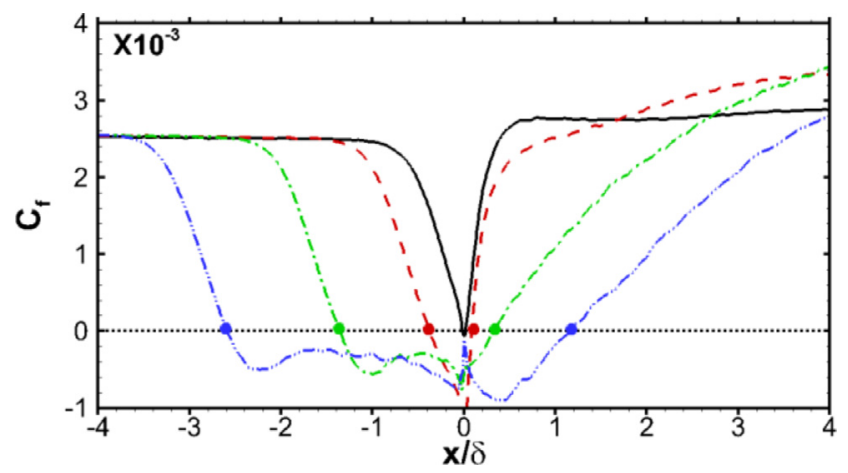

Fig. 15. Distribution of the mean wall skin-friction coefficient for different turning angles.

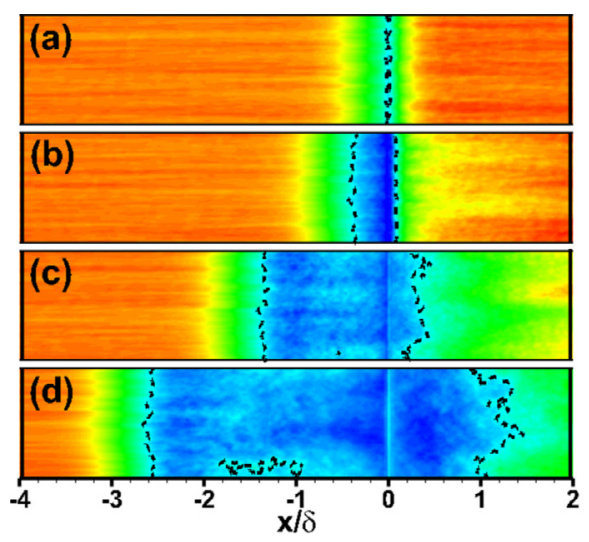

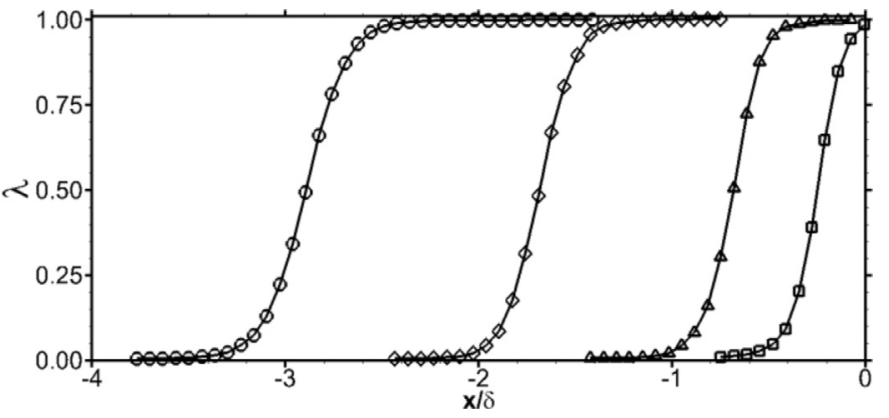

Fig. 17. Distribution of intermittency factor. Open circle: $24^{\circ}$; diamond: $20^{\circ}$; triangles: $14^{\circ}$; square: $8^{\circ}$.

boundary layer. However, at the mean separation point and in the separation bubble, the characteristic frequencies are much lower, with the peak frequency equal to $0.008 \mathrm{U}_{\infty} / \delta$. This result is in good agreement with the previous conclusion $[12,27]$. Furthermore, five disturbance frequencies, which are added in the flat-plate region, are also plotted in Fig. 10. It is obvious that the disturbance frequencies are concentrated on the high range of spectrum.

\section{Results and discussion}

\subsection{Flow visualizations}

Fig. 11 shows instantaneous temperature in the midspan section $(\mathrm{z}=7 \mathrm{~mm})$, which also plots the transition process in the flat-plate region. The entire process of transition to turbulence and the

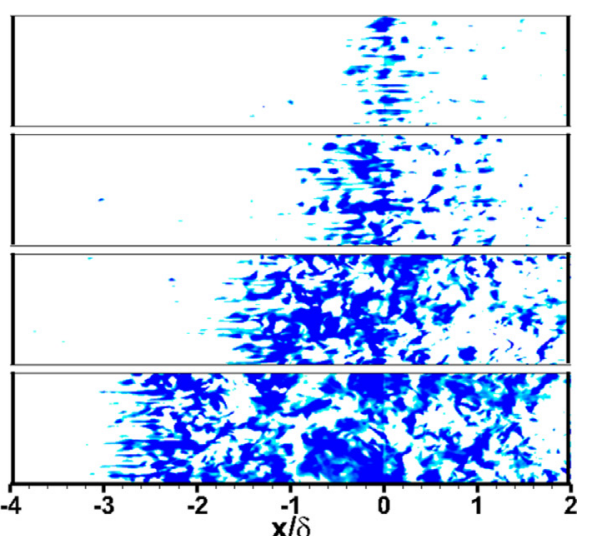

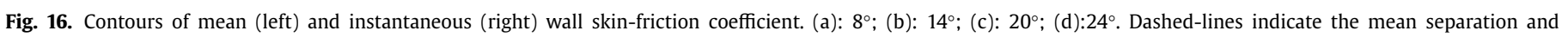
reattachment point, respectively. 

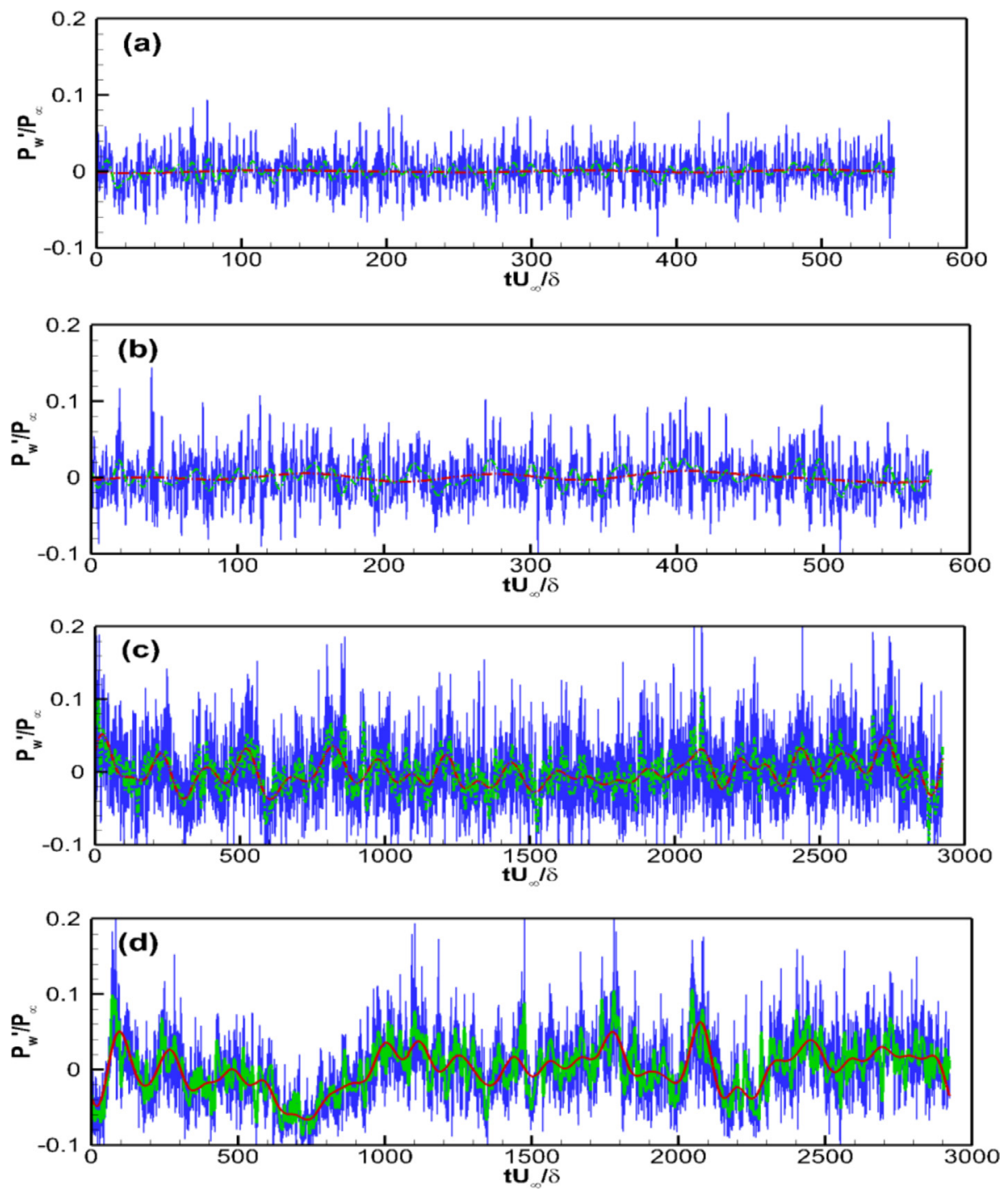

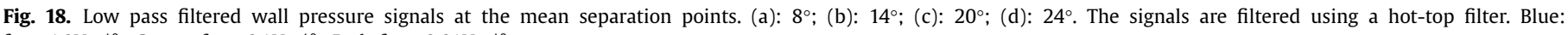
$\mathrm{f}_{\text {cut }}=1.0 \mathrm{U}_{\infty} / \delta ;$ Green: $\mathrm{f}_{\text {cut }}=0.1 \mathrm{U}_{\infty} / \delta ;$ Red: $\mathrm{f}_{\text {cut }}=0.01 \mathrm{U}_{\infty} / \delta$.

appearance of turbulence bursts at the onset of transition followed by a fully developed region $(\boldsymbol{x} \geq-60 \mathrm{~mm})$ are apparent. The fully developed turbulent boundary layer is characterized by turbulent bulges, exhibiting a highly intermittent character. As the turning angle is increased, the shock strength increases and the streamwise location of shock wave shifts toward upstream. Additionally, compared with upstream turbulent boundary layer, the high temperature regions in the downstream region become more pronounced.

Fig. 12 plots the instantaneous numerical schlieren in $8^{\circ}$ and $24^{\circ}$ cases. For a better display, the variable is defined as [12]

$N_{S}=0.8 \exp \left[-10\left(|\nabla \rho|-|\nabla \rho|_{\text {min }}\right) /\left(|\nabla \rho|_{\max }-|\nabla \rho|_{\min }\right)\right]$

It is shown that the foot of main shock penetrates into the turbulent boundary layer. Turbulence fluctuation is amplified greatly by the interaction with shock wave. Furthermore, shock wave in the $24^{\circ}$ case is deformed by the traveling shocklets, extending out from the edge of boundary layer downstream the interaction region. However, in the $8^{\circ}$ case, the shock is much closer to ramp surface and there is no apparent shocklets.

\subsection{Analysis of separation bubble}

Simpson [28] defines a statistical quantity to classify the separation. The probability of reversed flow $\gamma_{\mathrm{u}}$, which is computed by the fraction of total time that the instantaneous backflow happens, is used as the criterion. According to the definition of Simpson [28], incipient detachment (ID) occurs at $\gamma_{\mathrm{u}}=0.01$, Intermittent transitory detachment (ITD) occurs at $\gamma_{\mathrm{u}}=0.2$, transitory detachment (TD) occurs at $\gamma_{\mathrm{u}}=0.5$. The streamwise location where $\gamma_{\mathrm{u}}$ $=0.5$ indicates the occurrence of mean flow reverse.

The distribution of $\gamma_{\mathrm{u}}$ is plotted in Fig. 13. It is clear that the size of separation bubble increases as the turning angle increases. The effect of turning angle on separation bubble is further illustrated in Fig. 14, which plots the probability distribution of wall points with separation. The results indicate that all the four cases are the mean separated flow. The separation region in the $8^{\circ}$ case is rather small. But it is interesting to note that the streamwise distribution of separation and reattachment point appears to be symmetric. As the turning angle is increased, the separation point moves upstream and the reattachment point moves downstream. Moreover, the separation regions of $14^{\circ}, 20^{\circ}$ and $24^{\circ}$ cases are asymmetric. 

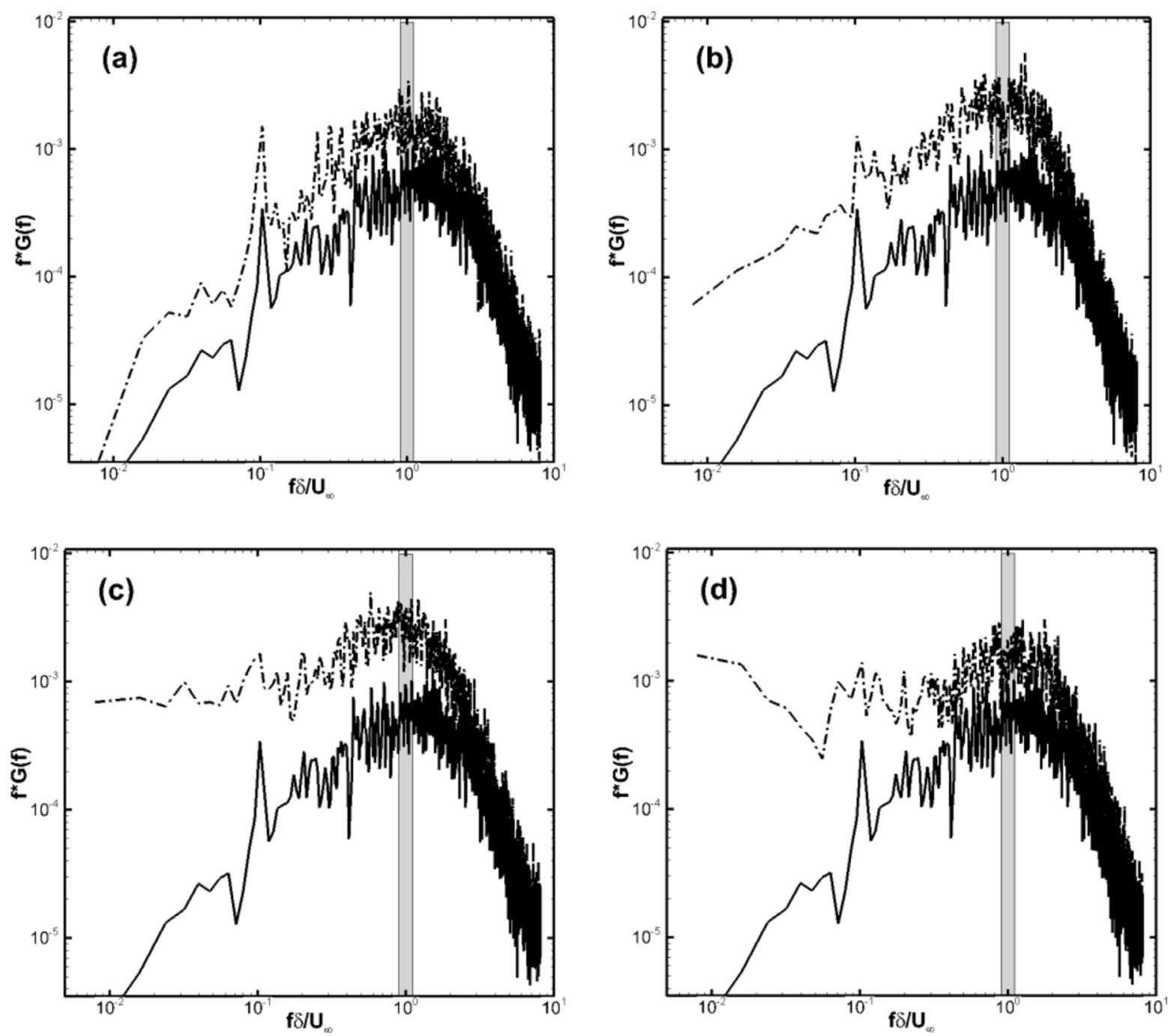

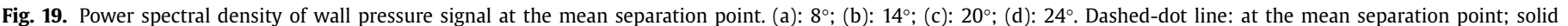
line: at $\mathrm{x}=-5.4 \delta$.

Fig. 15 plots the streamwise distribution of the mean wall skin-friction coefficient. The separation and reattachment point are defined as the point where the skin-friction coefficient changes sign. Therefore, the region with $C_{f} \leq 0$ indicates the separation region. It is found that the location of separation and reattachment, which is labeled as circles in Fig. 15, is consistent with the probability of reversed flow.

To further illustrate the intermittency and three dimensionality of separation bubble in the interaction region, the time averaged skin-friction coefficient contour for the four cases are shown in Fig. 16(a). For better comparison, locations of the mean separation and reattachment points are also labeled by black dashed vertical lines. It is apparent that the separation regions are not continuous and distributed non-uniformly, especially at the reattachment region in the $20^{\circ}$ and $24^{\circ}$ cases. Additionally, Fig. 16 (b) plots an instantaneous skin-friction coefficient contour. It is evident that the size of instantaneous separation region is much larger than the time-averaged result. These observations confirm that the separation bubble is highly intermittent and three-dimensional.

\subsection{Unsteady motion of shock wave}

In order to study the unsteady motion of shock wave, statistical quantities of wall pressure fluctuations are used to characterize the unsteadiness. The streamwise length scale of shock motion can be estimated by the intermittency factor, which is defined as [29]

$\lambda=\frac{\operatorname{time}\left[P_{w}>\left(\left\langle P_{w I}\right\rangle+3 \sigma\left(P_{w I}\right)\right)\right]}{\text { totaltime }}$

where $\mathrm{P}_{\mathrm{w}}$ represents the instantaneous wall pressure, $\left\langle\mathrm{P}_{\mathrm{wI}}\right\rangle$ and $\sigma\left(\mathrm{P}_{\mathrm{wI}}\right)$ represent the time-averaged wall pressure and the corresponding standard deviation in the upstream undisturbed turbulent boundary layer, respectively.

Fig. 17 plots the distribution of intermittency factor. It is clear that the profile shifts away from the corner region as the turning angle increases. However, the profile shape is not affected by the turning angle. In this paper, the streamwise length scale of shock motion is calculated as the length over which $0.04 \leq \lambda$ $\leq 0.98$. It is found that as the angle increases, the length scale increases linearly from $0.48 \delta$ in the $8^{\circ}$ case to $0.73 \delta$ in the $24^{\circ}$ case, which is consistent with the tendency of high Reynolds number experiments [3].

A hot-top filter defined as $\hat{P}^{\prime}(f)=G(f) P^{\prime}(f)$ is applied to the raw signals, where $P^{\prime}(f)$ is the spectrum of pressure fluctuations and $\hat{P}^{\prime}(f)$ is the filtered pressure signal in spectral space. The filter function $G(f)$ is defined as

$G(f)= \begin{cases}1 & f \leq f_{\text {cut }} \\ 0 & f>f_{\text {cut }}\end{cases}$ 


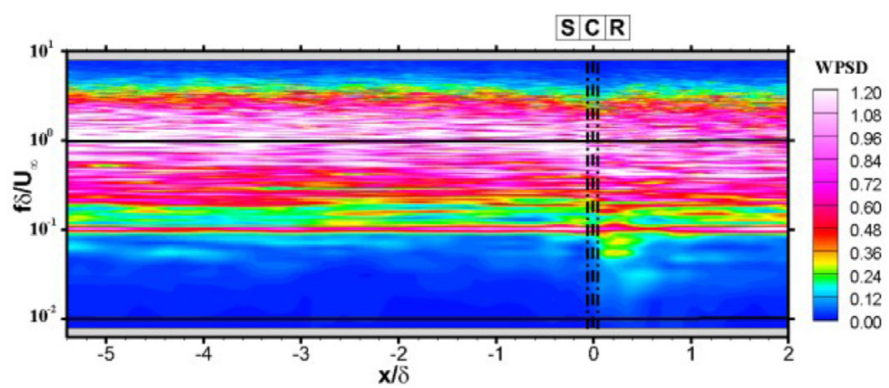

(a) $8^{\circ}$

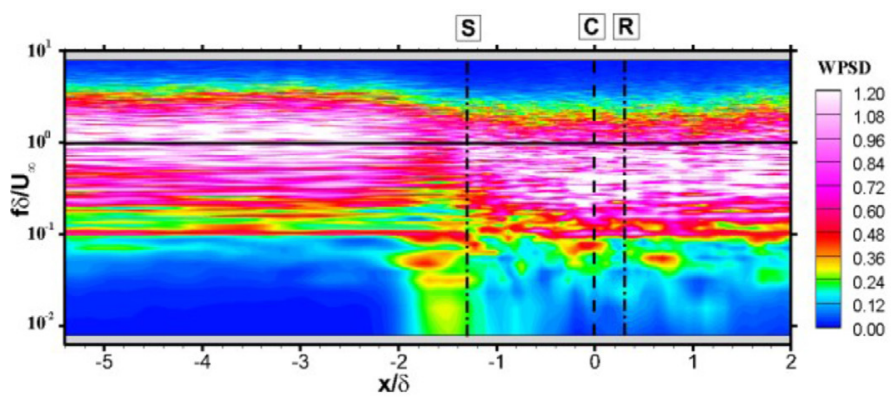

(c) $20^{\circ}$

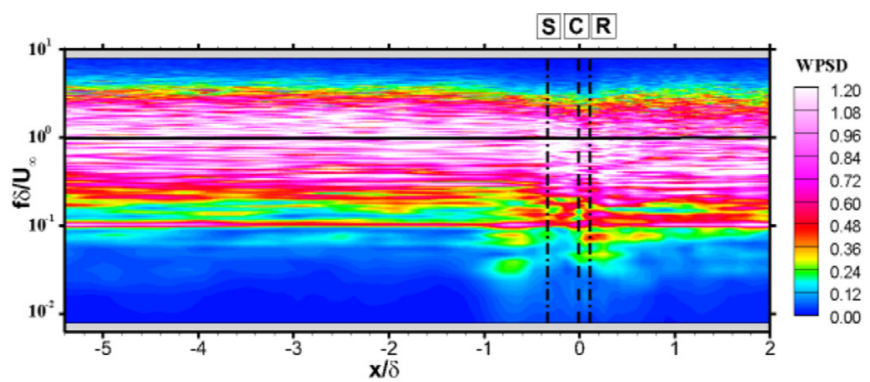

(b) $14^{\circ}$

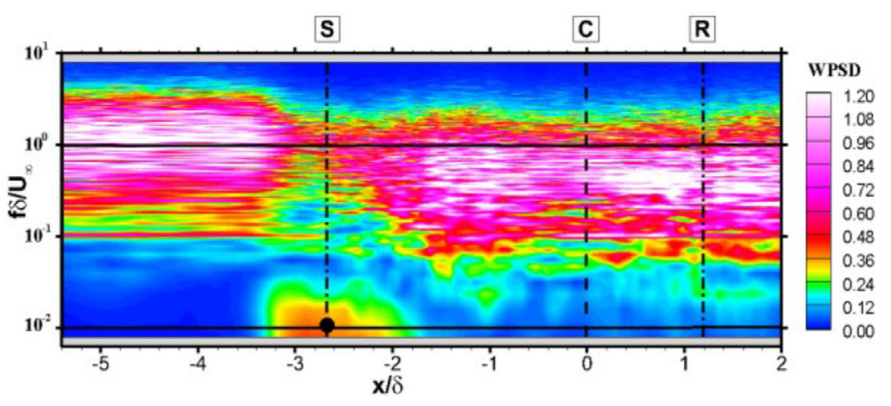

(d) $24^{\circ}$

Fig. 20. Contours of the weighted power spectral density of wall pressure signals. Dash-dotted lines indicate the mean separation and reattachment point, respectively.
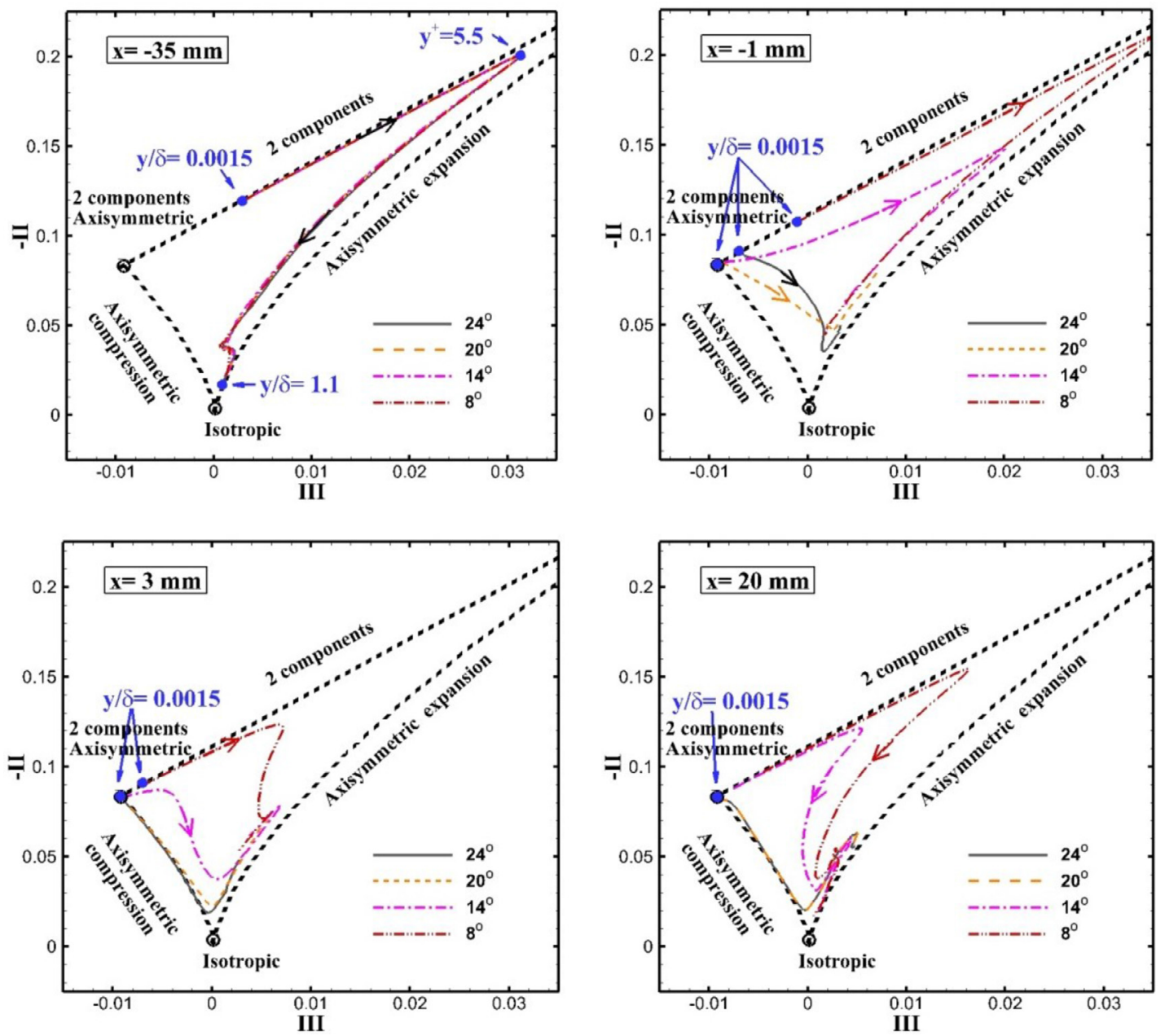

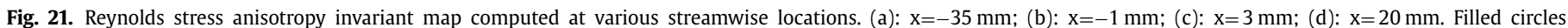
denote the wall point and arrows indicate the wall-normal direction. 


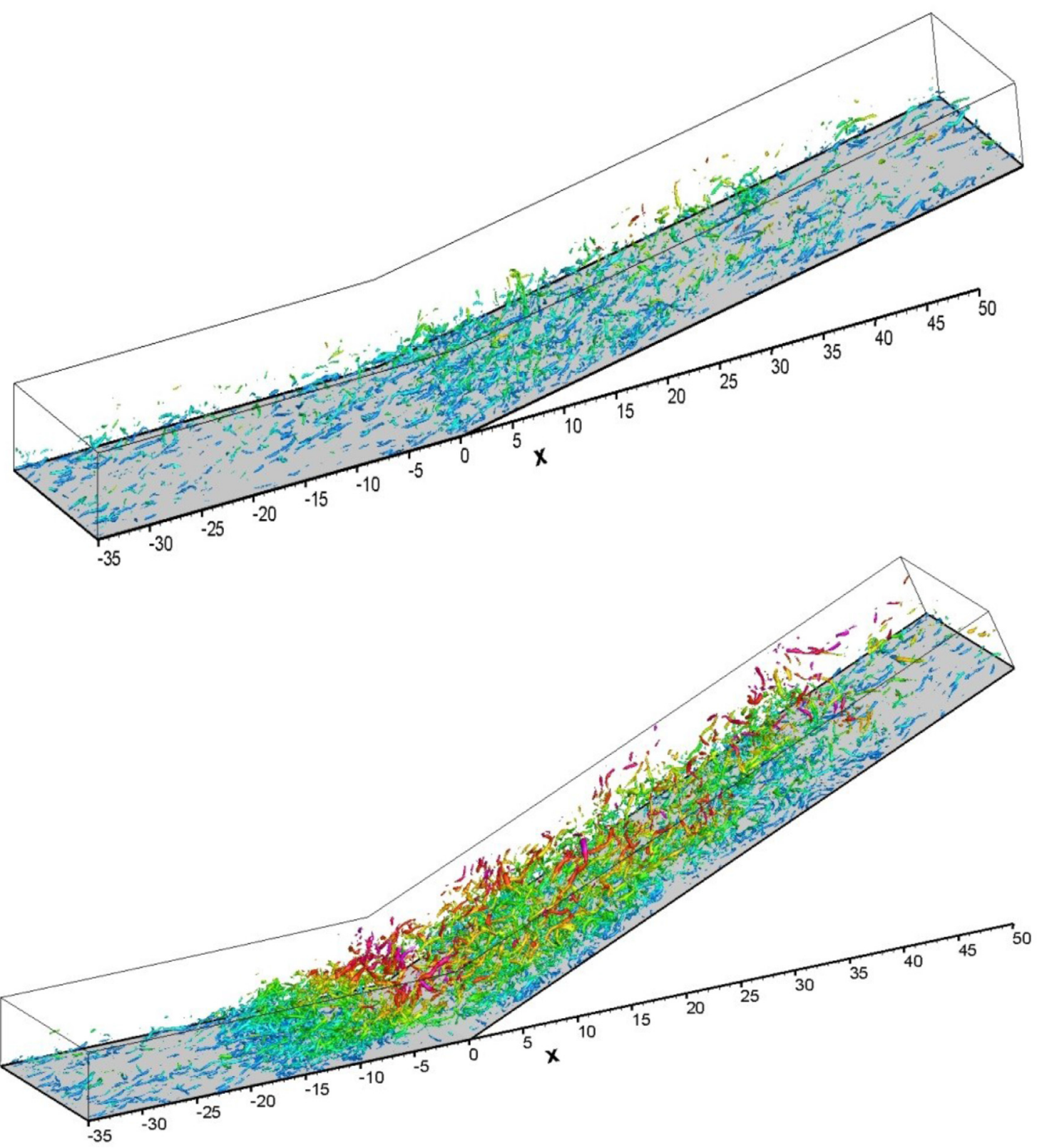

Fig. 22. Isosurface of the second invariant of velocity gradient tensor, colored by wall-normal distance. Top: $8^{\circ}$; bottom: $24^{\circ}$.

Thus, the fluctuations below cutoff frequency $\left(f_{\text {cut }}\right)$ can pass the filter and others are cut off.

Fig. 18 plots the low-pass filtered wall pressure signals at the mean separation point. Three different cutoff frequencies are used, which are $1.0 \mathrm{U}_{\infty} / \delta, 0.1 \mathrm{U}_{\infty} / \delta$ and $0.01 \mathrm{U}_{\infty} / \delta$. It is clear that the signals show a wide range of frequency. Obviously, high frequency fluctuations play the leading role in the $8^{\circ}$ and $14^{\circ}$ cases. The signals in the $20^{\circ}$ and $24^{\circ}$ cases are characteristic of low frequency. Additionally, magnitude of fluctuations in the $20^{\circ}$ and $24^{\circ}$ cases is larger than that of $8^{\circ}$ and $14^{\circ}$ cases. The corresponding power spectral density of wall pressure signals is plotted in Fig. 19. For the sake of contrast, the spectra at $\mathrm{x}=-5.4 \delta$ is also displayed together. It is found that the spectra in the $8^{\circ}$ and $14^{\circ}$ cases are similar. A bump centered about $1.0 U_{\infty} / \delta$ is observed. Furthermore, no significant energy is present below the frequency of $0.01 \mathrm{U}_{\infty} / \delta$. As the turning angle increases, the energy at high frequency shows little change. But the energy at low frequency increases significantly. It is also suggested that the unsteady shock motion in the $20^{\circ}$ and $24^{\circ}$ cases is characterized by the low frequency.

To further illustrate the unsteadiness of interaction, contour of weighted power spectral density (WPSD) is plotted in Fig. 20, which is as function of frequency and streamwise location. The mean separation point and reattachment point are also plotted to indicate separation region. It is apparent that the separation regions in the $8^{\circ}$ and $14^{\circ}$ cases are rather small and the dominant frequency maintains at the characteristic frequency of incoming turbulent boundary layer. The separation regions in the $20^{\circ}$ and $24^{\circ}$ cases increase dramatically. And the behavior of wall pressure fluctuations through the interaction is utterly different from that of small turning angles. It is apparent that the spectra are centered at $1.0 \mathrm{U}_{\infty} / \delta$ in the incoming turbulent boundary. Subsequently, the dominant frequency in separation region shifts from $1.0 \mathrm{U}_{\infty} / \delta$ to $0.01 U_{\infty} / \delta$. Downstream of the interaction region, most energetic frequency recovers back to $1.0 \mathrm{U}_{\infty} / \delta$. This behavior agrees well with previous numerical and experimental studies [14]. 


\subsection{Reynolds stress anisotropy tensor}

The turbulence state through the interaction region can be characterized by the change in anisotropy of Reynolds stress tensor. The invariants of Reynolds stress anisotropy tensor is defined as [30]

$b_{i j}=\frac{<\rho u^{\prime \prime}{ }_{i} u^{\prime \prime}{ }_{j}>}{2<\rho><k>}-\frac{1}{3} \delta_{i j}$

A representative picture of turbulence state is shown by its second and third invariants

$I I_{b}=b_{i j} b_{j i} \quad I I I_{b}=b_{i j} b_{j k} b_{k i}$

As pointed out by Lumley [30], all realizable flow-fields must lie inside the anisotropy invariant map (also called as Lumley triangle). The lines and vertices are corresponding to special states of turbulence.

Fig. 21 shows the anisotropy invariant maps for selected streamwise locations. In the upstream undisturbed turbulent boundary layer, a two-component turbulence state is observed near the wall. At the outer layer of boundary layer, the turbulence is close to isotropic status. The maximum anisotropy of turbulence occurs at $\mathrm{y}^{+}=5.5$, which is representative for the typical elongated streaky structure with alternating low and high momentum fluids. These observations are in good agreement with Pirozzoli et al. [31] and Grilli et al. [32]. The behaviors of turbulence state in the interaction region are shown in Fig. 21(b) and (c). As the turning angle is increased, turbulence in the vicinity of the wall approach is gradually approaching the two-component axisymmetric state. Moreover, turbulence states in the inner part of boundary layer approach closer to the axisymmetric compression line. However, in the outer region of boundary layer, turbulence evolves towards an axisymmetric expansion state. Fig. 21d plots the turbulence state after the reattachment point. The increase of anisotropy in the near wall region of $8^{\circ}$ and $14^{\circ}$ shows a reversal tendency, indicating that the flow rapidly recovers to the initial streaky structure.

\subsection{Coherent structure}

For a deeper understanding of the evolution of turbulent boundary layer in the interaction region, the instantaneous coherent vortex structure is studied through the second invariant of velocity gradient tensor $\mathbf{Q}$ which is defined as [33]

$$
\begin{aligned}
Q & =\frac{1}{2}\left(\tilde{\Omega}_{i j} \tilde{\Omega}_{i j}-\tilde{S}_{i j} \tilde{S}_{i j}\right) \\
\tilde{\Omega}_{i j} & =\frac{1}{2}\left(\frac{\partial \bar{u}_{i}}{\partial x_{j}}-\frac{\partial \bar{u}_{j}}{\partial x_{i}}\right) \quad \tilde{S}_{i j}=\frac{1}{2}\left(\frac{\partial \bar{u}_{i}}{\partial x_{j}}+\frac{\partial \bar{u}_{j}}{\partial x_{i}}\right)
\end{aligned}
$$

The variables $\tilde{\Omega}_{i j}$ and $\tilde{S}_{i j}$ denote the symmetric and asymmetric part of velocity gradient tensor, respectively. The presence of a vortex is decided by whether the local rate of rotation is larger than the rate of strain or not.

The instantaneous vortex structures are clearly visible in Fig. 22. It plots an isosurface of the second invariant of velocity gradient tensor, which is colored by the wall-normal distance. It is apparent that the structures between $8^{\circ}$ and $24^{\circ}$ cases are significantly different. In the small turning angle, the cane-like streamwise vortexes in the near-wall region are the dominant structure. However, in the large turning angle, the large-scale hairpin vortexes and packets in the outer layer play the leading role, especially in the separated shear layer and reattachment region.

Fig.. 23 depicts the instantaneous streamwise velocity in an $x-z$ plane parallel to the wall. It is apparent that the velocity field of incoming turbulent boundary layer exhibits a typical elongated streaky structure with alternating low and high momentum regions. In the separation region, this canonical structure

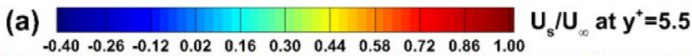

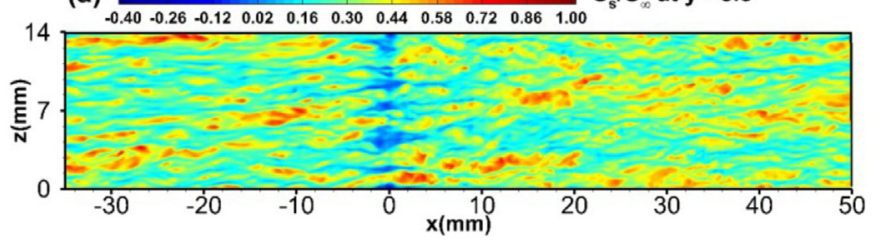

(b)

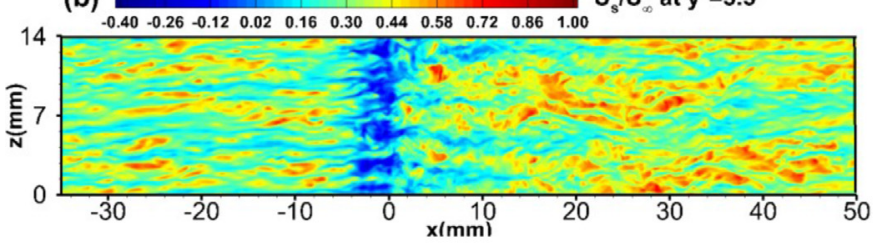

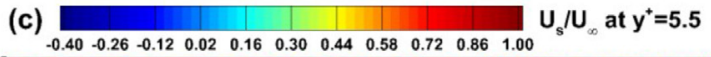

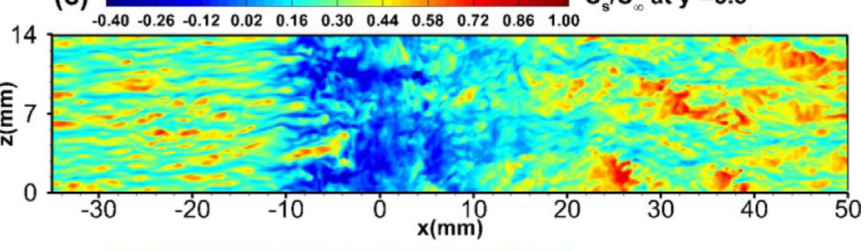

(d) $U_{s} / U_{\infty}$ at $y^{+}=5.5$

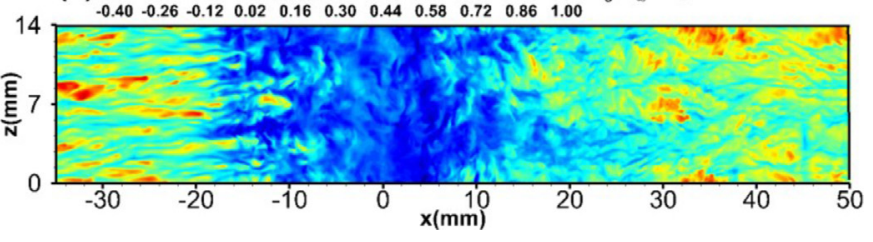

Fig. 23. Instantaneous streamwise velocity contours in plane $x-z$ at $y^{+}=5.5$. (a): $8^{\circ}$; (b): $14^{\circ} ;$ (c): $20^{\circ}$; (d): $24^{\circ}$.

Table 2

\begin{tabular}{|c|c|}
\hline Expression & Meanings \\
\hline$K=1 / 2 \overline{\rho u^{\prime \prime}{ }_{i} u^{\prime \prime}}{ }_{i} / \bar{\rho}$ & Turbulent kinetic energy \\
\hline$C=-\partial \bar{\rho} \tilde{u}_{j} k / \partial x_{j}$ & Convection \\
\hline$P=-\overline{\rho u_{i}^{\prime \prime}{ }_{i}^{\prime \prime}{ }_{j}} \partial \tilde{u}_{i} / \partial x_{j}$ & Production \\
\hline$T=-\partial\left(1 / 2 \overline{\rho u^{\prime \prime}{ }_{i} u^{\prime \prime}{ }_{i} u^{\prime \prime}{ }_{j}}+\overline{p^{\prime} u^{\prime \prime}}{ }_{j}\right) / \partial x_{j}$ & Turbulent transport \\
\hline$V=\partial \overline{\sigma_{i j}{ }_{i j}{ }^{\prime \prime}}{ }_{i} / \partial x_{j}$ & Viscous diffusion \\
\hline$D=\overline{\sigma^{\prime}{ }_{i j} \partial u^{\prime \prime}{ }_{i} / \partial x_{j}}$ & Dissipation \\
\hline$K=\overline{p^{\prime} \partial u^{\prime \prime}{ }_{i} / \partial x_{i}}$ & Pressure dilation \\
\hline$M=\overline{u^{\prime \prime}}{ }_{i}\left(\partial \overline{\sigma_{i j}} / \partial x_{j}-\partial \bar{p} / \partial x_{i}\right)$ & Mass diffusion \\
\hline
\end{tabular}

Explicit expressions for the terms in TKE budget equation.

is badly destroyed by the interaction with shock wave and the flow becomes chaotic and isotropic. The initial streaky structure is recovered after the interaction region. Furthermore, it is obvious that the recovery of streaky structures in $8^{\circ}$ and $14^{\circ}$ is much faster than the other two cases. This observation also further confirms the above analysis of Reynolds stress anisotropy tensor.

\subsection{Turbulent kinetic energy budgets}

Analysis of turbulent kinetic energy (TKE) budgets in compressible flow is of great help in understanding the turbulence structure and improving the existing turbulence models. The compressible turbulent kinetic energy transport equation is in the form as [34]

$\frac{\partial}{\partial t}(\bar{\rho} k)=C+P+T+V-D+K+M$

The explicit expressions of all terms in above equation are shown in Table 2 . In this paper, the production term $P$, turbulent 

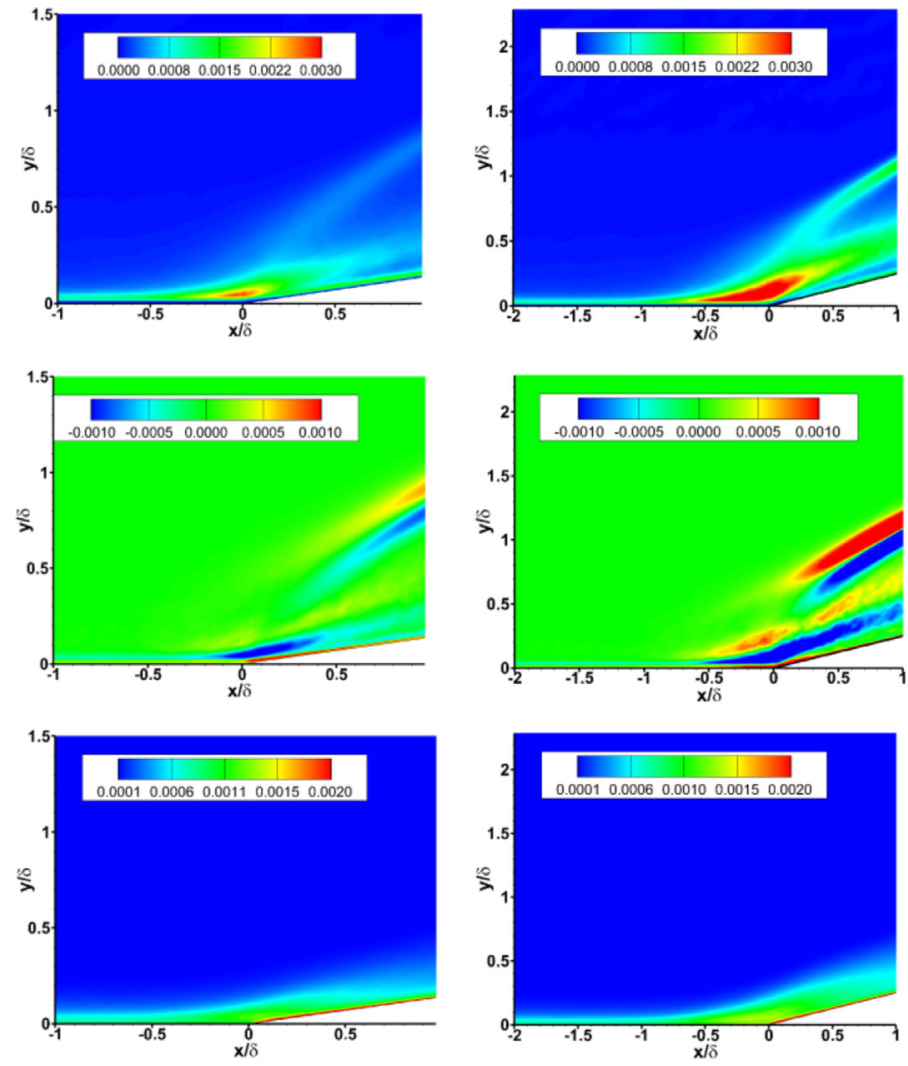

(a) $8^{\circ}$

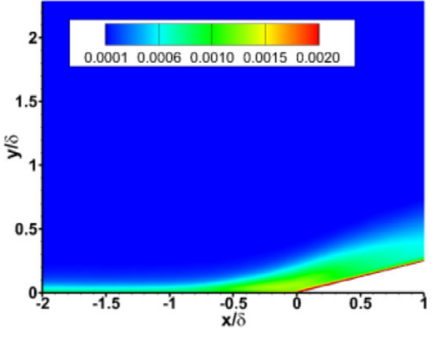

(b) $14^{\circ}$
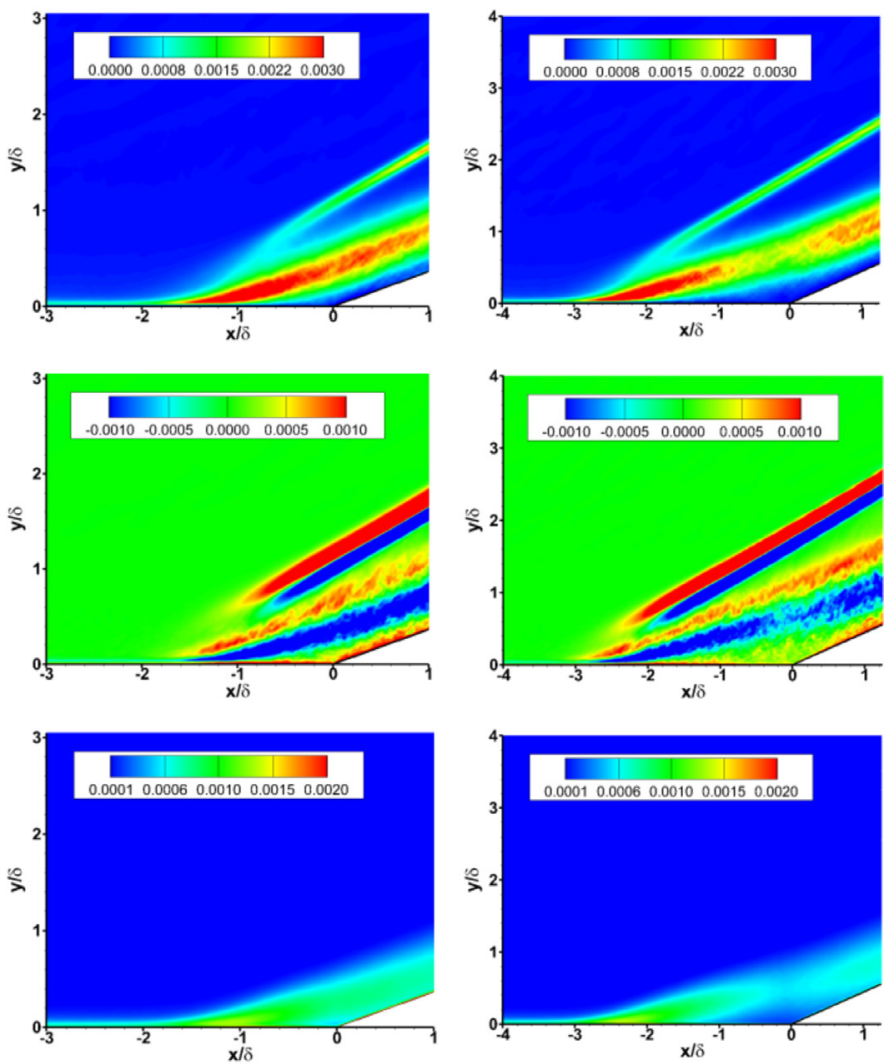

(c) $20^{\circ}$

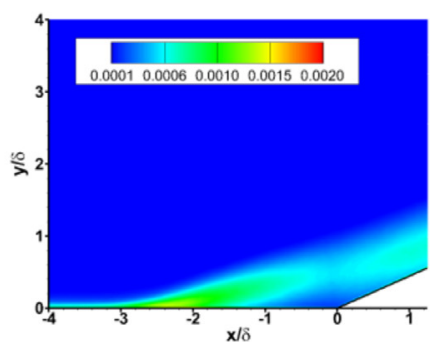

(d) $24^{0}$

Fig. 24. Contours of main terms in turbulence kinetic energy budgets. From top to bottom: production term $P$, turbulent transport term $T$ and dissipation term $D$.

transport term $T$, viscous diffusion term $V$ and dissipation term $D$ are the emphases of research.

Fig. 24 plots the two dimensional distribution of production term, turbulent transport term and dissipation term in the corner region. It is apparent that the peak value of production term is observed in the region of separated shear layer above separation bubble and in the region of shock wave. This is due to the strong mean shear around these regions [15]. Moreover, as the turning angle increases, the region with high value of production term increases significantly, especially in the region of shear layer. Obviously, the turbulent transport term is also mainly distributed in the shock wave and the shear layer. As shown in Fig. 24, the distribution is characteristic of two-layer structures with negative and positive values. Additionally, the magnitude along separation shock-wave is much larger than that of shear layer, especially in the $20^{\circ}$ and $24^{\circ}$ cases. The dissipation term at the near-wall region is strong for all cases, but the location of peak value shows significant difference as the turning angle increases. The peak value of dissipation term in the $8^{\circ}$ and $14^{\circ}$ cases is present downstream the interaction region. However, in the $20^{\circ}$ and $24^{\circ}$ cases, the peak value is observed in the foot of separated shear layer.

In order to further illustrate the transport mechanism, Fig. 25 shows the one-dimensional profiles of TKE budgets at $x=0 \mathrm{~mm}$, as a function of wall distance normalized by boundary layer thickness at the reference point. According to the above analysis of flow-field, the selected location is in the separation bubble for all cases. It is shown in Fig. 25(a) and (b) that the shape of profiles is similar. The peak value of production and dissipation term occurs in the near-wall region. Turbulent transport and viscous diffusion become significant in the inner part of the boundary layer, which transports the turbulent kinetic energy from far-from-wall
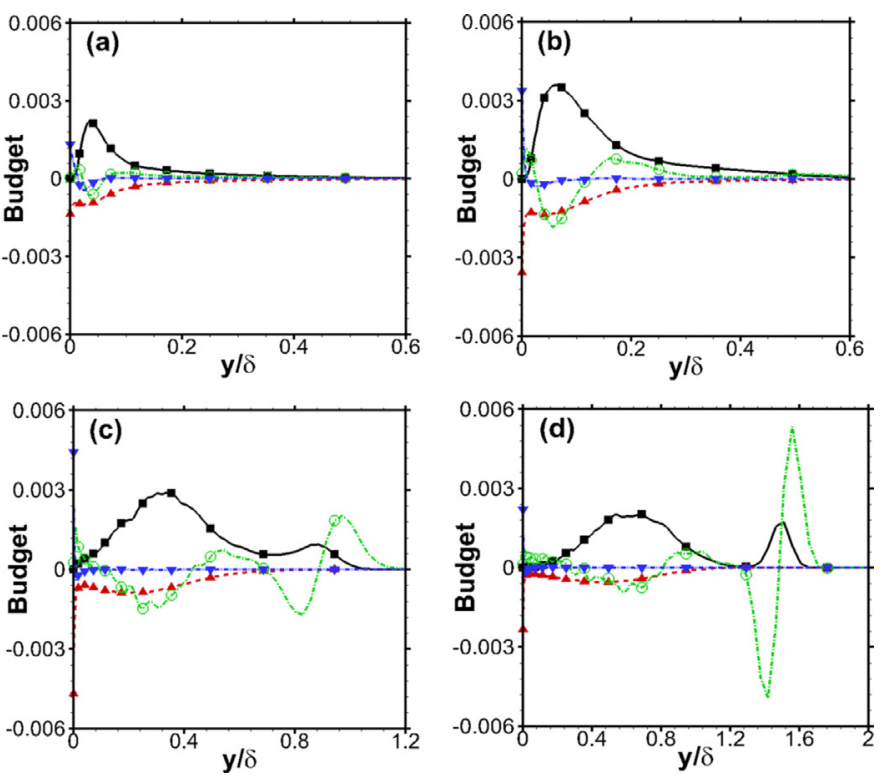

Fig. 25. Turbulence kinetic energy budget at $\mathrm{x}=0 \mathrm{~mm}$ in function of $\mathrm{y} / \delta$. (a): $8 \mathrm{deg}$; (b): $14 \mathrm{deg}$; (c): $20 \mathrm{deg}$; (d):24 deg. Symbol: $\mathbf{\square}$, production; ॰, turbulent transport; $\mathbf{\nabla}$, viscous diffusion; $\boldsymbol{\Lambda}$, dissipation.

region to the near-wall region to be dissipated. The mechanism is likewise that of canonical flat-plate turbulent boundary layer [15].

A qualitatively different behavior is observed in Fig. 25(c) and (d). The distribution of turbulent production term in $20^{\circ}$ and $24^{\circ}$ cases is characteristic of two peaks, with one peak above the 
separation bubble and the other near the shock wave. The peak value of dissipation term and viscous diffusion is also observed in the near-wall region, which is similar to that of $8^{\circ}$ and $14^{\circ}$ cases. However, compared with the viscous diffusion term, the turbulent transport terms show significant changes across the separation bubble and shock region. It suggests that only the turbulent transport term plays a major role in balancing turbulent production and dissipation terms.

\section{Conclusions}

In this paper, direct numerical simulations are performed to study the interactions of shock waves with a supersonic turbulent boundary layer in compression corners with different turning angle. Accuracies of this DNS are validated by comparison with pervious simulation and experimental data, and the effects of turning angle are evaluated.

(1) The computation results agree well with previous experimental and DNS data in the mean wall pressure, skin-friction coefficient, as well as velocity profile and turbulence intensity.

(2) With the turning angle increasing, the strength of shock wave and three-dimensional behavior of separation bubble are both dramatically enhanced. The characteristic properties of unsteady shock motion in the $8^{\circ}$ and $14^{\circ}$ cases are the small-scale and high frequency oscillations. Furthermore, the low frequency and large-scale oscillations of shock motion in the $20^{\circ}$ and $24^{\circ}$ cases has strong relation with the length scale of separation bubble in ramp corner.

(3) The coherent vortex structures and anisotropy of Reynolds stress tensor at interaction region of various turning angles are different. With the increment of the turning angle, large-scale hairpin vortexes and packets in the outer of boundary layer gradually play the major role. The canonical streaky structures in turbulent boundary layer are destroyed dramatically by the interactions, especially in the $20^{\circ}$ and $24^{\circ}$ cases.

(4) Turning angle effect on the turbulent transport mechanism is significant at the corner region. In the $8^{\circ}$ and $14^{\circ}$ cases, the mechanism is similar with that of flat-plate turbulent boundary layer. In the $20^{\circ}$ and $24^{\circ}$ cases, the shear layers above separation bubble are critical for the transport mechanism.

\section{Acknowledgements}

These works is based on the research sponsored by the state key research and develop project (2016YFA0401200), the Science Challenge Project (JCKY2016212A501) and the National Natural Science Foundation of China (grants 91441103, 11372330, and 11472278). The authors thank National Supercomputer Center in Tianjin (NSCC-TJ), and National Supercomputer Center in GuangZhou (NSCC-GZ) for providing computer time.

\section{References}

[1] Ardonceau PL. The structure of turbulence in a supersonic shock wave/ boundary layer interaction. AIAA J 1984;22(9):1254-62.

[2] Smits AJ, Muck KC. Experimental study of three shock wave/turbulent boundary layer interactions. J Fluid Mech 1987;182:291-314.
[3] Dolling DS, Or CT. Unsteadiness of the shock wave structure in attached and separated compression flows. Exp Fluids 1985;3(1):24-32.

[4] Beresh SJ, Clemens NT, Dolling DS. Relationship between upstream turbulent boundary layer velocity fluctuations and separation shock unsteadiness. AIAA J 2002;40(12):2412-22.

[5] Erengil ME, Dolling DS. Unsteady wave structure near separation in a Mach 5 compression ramp interaction. AIAA J 1991;29(5):728-35.

[6] Ganapathisubramani B, Clemens NT, Dolling DS. Low frequency dynamics of shock-induced separation in a compression ramp interaction. J Fluid Mech 2009;636:397-425.

[7] Bookey PB, Wyckham C. New experimental data of STBLI at DNS/LES accessible Reynolds numbers. 35th AIAA Fluid Dynamics Conference and Exhibit Toronto, Ontario Canada; 2005. June 6-9No: 2005-4899.

[8] Touber E, Sandham ND. Comparison of three large-eddy simulations of shock-induced turbulent separation bubbles. Shock Waves 2009;19(1):469-78.

[9] Morgan B, Duraisamy K, Nguyen S. Flow physics and RANS modeling of oblique shock/turbulent boundary layer interaction. J Fluid Mech 2013;729:231-84.

[10] Rizzetta DP, Visbal MR. Application of large eddy simulation to supersonic compression ramps. AIAA J 2002;40(8):1574-81.

[11] Adams NA. Direct simulation of the turbulent boundary layer along a compression ramp at $M=3$ and $\operatorname{Re}_{\theta}=1685$. J Fluid Mech 2000;420:47-83.

[12] Wu M, Martin MP. Direct numerical simulation of supersonic turbulent boundary layer over a compression ramp. AIAA J 2007;45(4):879-89.

[13] Wu M, Martin MP. Analysis of shock motion in shock wave and turbulent boundary layer interaction using direct numerical simulation data. J Fluid Mech 2008;594:71-83.

[14] Priebe S, Wu M, Martin MP. Low-frequency unsteadiness in shock wave-turbulent boundary layer interaction. J Fluid Mech 2012;699:1-49.

[15] Li XL, Fu DX, Ma YW. Direct numerical simulation of shock/turbulent boundary layer interaction in a supersonic compression ramp. Sci China 2010;53(9):1651-8.

[16] Dolling DS. Fifty years of shock-wave boundary-layer interaction research: what next? AIAA J 2001;39(8):1517-30.

[17] Clemens NT, Narayanaswamy V. Low frequency unsteadiness of shock wave turbulent boundary layer interactions. Annu Rev Fluid Mech 2014;46:469-92.

[18] Gaitonde DV. Progress in shock wave boundary layer interactions. Prog Aerosp Sci 2015;72(1):80-99.

[19] Martin MP, Taylor EM, Wu M. A bandwidth optimized WENO scheme for the effective direct numerical simulation of compressible turbulence. J Comput Phys 2006;220(1):270-89.

[20] Li XL, Fu DX, Ma YW. Acoustic calculation for supersonic turbulent boundary flow. Chinese Phys Lett. 2009;26(1):094701.

[21] Gao H, Fu DX, Ma YW. Direct numerical simulation of supersonic turbulent boundary layer flow. Chinese Phys Lett. 2005;22(1):1709-12.

[22] Li XL, Fu DX, Ma YW. Direct numerical simulation of hypersonic boundary transition over a blunt cone with a small angle of attack. Phys Fluids 2010;22(1):025105.

[23] Pirozzoli S, Grasso F, Gatski TB. Direct numerical simulation and analysis of a spatially evolving supersonic turbulent boundary layer at $\mathrm{M}=2.25$. Phys Fluids 2004;16(1):530-45

[24] Wu X, Moin P. Direct numerical simulation of turbulence in a nominally zeropressure-gradient flat-plate boundary layer. J Fluid Mech 2009;630:5-41.

[25] Spalart PR. Direct numerical simulation of a turbulent boundary layer up to $\operatorname{Re}_{\theta}=1410$. J Fluid Mech 1988;187:61-98.

[26] Erm LP, Joubert J. Low Reynolds number turbulent boundary layers. J. Fluid Mech 1991;230:1-44.

[27] Dupont P, Piponniau S. Investigation by Particle Image velocimetry measurements of oblique shock reflection with separation. AIAA J 2008;46(6):1365-70.

[28] Simpson RL. Turbulent boundary layer separation. Annu Rev Fluid Mech 1989;21:205-34

[29] Loginov MS, Adams NA, Zheltovodov AA. Large-eddy simulation of shock wave turbulent boundary layer interaction. J Fluid Mech 2006;565:135-69.

[30] Lumley JL. Computational modeling of turbulent flows. Adv Appl Mech 1978;18(1):123-76.

[31] Pirozzoli S, Bernardini M, Grasso F. Direct numerical simulation of transonic shock/boundary layer interaction under conditions of incipient separation. J Fluid Mech 2010;657:361-93.

[32] Grilli M, Hichel S, Adams NA. Large-eddy simulation of a supersonic turbulent boundary layer over a compression-expansion ramp. Int J Heat Fluid Fl 2013;42(1):79-93.

[33] Jeong J, Hussain F. On the identification of a vortex. J Fluid Mech 1995;285:69-94.

[34] Pirozzoli S, Bernardini M. Direct numerical simulation database for impinging shock wave/turbulent boundary layer interaction. AIAA J 2011;49(6):1307-12.

[35] Xingkun Zhu, Changping Yu, Fulin Tong, Xinliang Li. Numerical study on wall temperature effects on shock wave/turbulent boundary-layer interaction. AIAA J 2017;55(1):131-40.

[36] White FM, Corfield I. Viscous fluid flow. New York: McGraw-Hill; 2006. 